SYNTHESIS, STRUCTURAL STABILITY CALCULATION AND ANTIBACTERIAL EVALUATION OF NOVEL 3,5 DIPHENYLCYCLOHEX-2-EN-1-ONE DERIVATIVES.

\author{
Omyma A. Abd Allah, ${ }^{1}$ Ahmed M. El-Saghier, ${ }^{1}$ and Asmaa M. Kadry ${ }^{1}$ \\ ${ }^{1}$ Chemistry Department, Faculty of Science, Sohag University, PO box 82524, Sohag, Egypt
}

Supplementary Materials 


\section{Synthesis of 5,7-diphenyl-2,6,7,7a-tetrahydrobenzo[d][1,2,3]thiadiazol-1-oxide (3).}

Freshly distilled thionyl chloride $(8 \mathrm{ml})$ was taken to a round bottom flask $(50 \mathrm{ml})$ placed in a salt-ice bath at about $-10{ }^{\circ} \mathrm{C}$, 3,5 -diphenylcyclohex2-en-1-semicarbazone (2) $(3.05 \mathrm{~g}, 0.01 \mathrm{~mol})$ was added in three portions maintaining temperature between $0{ }^{\circ} \mathrm{C}$ and $-10{ }^{\circ} \mathrm{C} . \quad$ After complete addition, the reaction mixture was allowed to stand at room temperature, $25 \mathrm{ml}$ of dichlomethane was added and the mixture was stirred for another 2 hrs. Excess thionyl chloride was decomposed by slowly adding sodium carbonate solution (15-20 ml) to the reaction mixture, the organic layer was separated, washed thoroughly with water,sodium bicarbonate (5\%) solution and dried over anhydrous sodium sulphate. The solvent was evaporated under reduced pressure, the separated was filtered off and crystallized from water as pale green fine powder; Yield: 52\%; mp 133-135 ${ }^{\circ}$; IR $\left(\lambda \max , \mathrm{cm}^{-1}\right): 3462(\mathrm{NH}), 3041\left(\mathrm{CH}_{\text {arom }}\right), 2945,2896\left(\mathrm{CH}_{\text {aliphatic }}\right), 1659(\mathrm{C}=\mathrm{N}), 1262(\mathrm{C}-\mathrm{S}), 1019(\mathrm{~S}=0) ;{ }^{1} \mathrm{H}-\mathrm{NMR}\left(\mathrm{DMSO}-\mathrm{d}_{6}\right), \delta$ ppm :7.70-7.26(m, 10H, $\left.\mathrm{CH}_{\text {arom. }}\right), 6.47\left(\mathrm{~s}, 1 \mathrm{H}, \mathrm{CH}_{\text {oliph. }}\right), 3.45-3.44\left(\mathrm{~d}, 2 \mathrm{H}, \mathrm{CH}_{2}\right), 3.02\left(\mathrm{~s}, 2 \mathrm{H}, 2 \mathrm{NH}\right.$, disappeared by $\left.\mathrm{D}_{2} \mathrm{O}\right), 2.80\left(\mathrm{t}, 1 \mathrm{H}, \mathrm{CH}-\mathrm{Ph}\right.$.); ${ }^{13} \mathrm{C}-$ NMR (DMSO-d $\left.)_{6}\right), \delta$ ppm: 35.74( $\left(\mathrm{CH}_{2}\right), 44.06(\mathrm{CH}-\mathrm{Ph}), 124.67\left(\mathrm{CH}_{\text {oliph. }}\right), 126.82,127.13,127.54,128.97,129.27,136.58,138.51(\mathrm{C}$ of $\mathrm{Ph}$ rings $), 144.21$

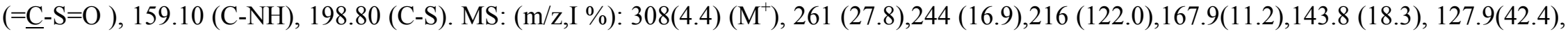
107.6(16.3), 91.1(100.0), 77.9(24.4), 62.0(10.2), 49.8(16.6), calc. for $\mathrm{C}_{18} \mathrm{H}_{16} \mathrm{~N}_{2} \mathrm{OS}$ (M.wt.= 308.3). 


\section{Synthesis of 3-carbaldehyde-4,6-diphenyl-3,3a,4,5-tetrahydro-2H-indazole (4)}

A mixture of DMF (2.3 ml, 0.3 mole) and $\mathrm{POCl}_{3}(2.8 \mathrm{ml}, 0.3 \mathrm{~mole})$ was stirred at $0{ }^{\circ} \mathrm{C}$ for $10 \mathrm{~min}$, a solution of the 3,5-diphenylcyclohex-2-en-1semicarbazone (2) $(3.05 \mathrm{~g}, 0.1 \mathrm{~mol})$ in DMF $(10 \mathrm{ml})$ was added dropwise. The reaction mixture was stirred at room temperature for $1 \mathrm{hrs}$. and then heated at $70{ }^{\circ} \mathrm{C}$ for $4 \mathrm{hrs}$. After cooling at room temperature, the mixture was neutralized with cold potassium carbonate solution. The separated solid was filtered off, washed with water, dried and crystallized from ethanol as yellow crystal; Yield: $47 \%$; $\mathrm{mp} 169-170^{\circ} \mathrm{C} ; \mathrm{IR}\left(\lambda \mathrm{max}, \mathrm{cm}^{-1}\right)$ : 3454,3403(NH), 3041( $\left.\mathrm{CH}_{\text {arom }}\right), 2927\left(\mathrm{CH}_{\text {aliphatic }}\right), 1682(\mathrm{C}=\mathrm{O}), 1573(\mathrm{C}=\mathrm{C}), 1262(\mathrm{C}-\mathrm{S}), 1107(\mathrm{~S}=\mathrm{O}) ;{ }^{1} \mathrm{H}-\mathrm{NMR}\left(\mathrm{DMSO}-\mathrm{d}_{6}\right), \delta$ ppm: $9.35(\mathrm{~s}, 1 \mathrm{H}$, $\mathrm{CHO}), 7.72-7.24\left(\mathrm{~m}, 10 \mathrm{H}, \mathrm{CH}\right.$ arom.),6.67(s,1H, $\left.\mathrm{CH}_{\text {oliph.. }}\right), 6.46,6.34(\mathrm{~s}, 2 \mathrm{H}, 2 \mathrm{NH}), 5.86(\mathrm{~m}, 1 \mathrm{H}, \underline{\mathrm{CHPh}}), 3.15(\mathrm{~m}, 1 \mathrm{H}, \underline{\mathrm{CH}}-\mathrm{CHCHO}), 3.03-3.01(\mathrm{~d}, 1 \mathrm{H}, \underline{\mathrm{CH}}-$

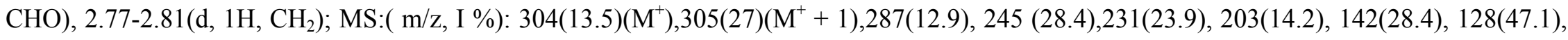
115(74.2), 103(38.1), 91(100), 78 (27.1), calc. for $\mathrm{C}_{20} \mathrm{H}_{20} \mathrm{~N}_{2} \mathrm{O}($ M. wt. $=304.3)$.

\section{Synthesis of 5,7-diphenyl-6,7-dihydro-2(3H)-imino-1,3-benzo[d]thiazol (5).}

To a solution of 3,5-diphenylcyclohex-2-en-1-one (1) (0.39 g, $0.0015 \mathrm{~mol})$ and thiourea $(0.11 \mathrm{~g}, 0.0015 \mathrm{~mol})$ in isopropanol (40 ml), iodine (0.25 g, $0.002 \mathrm{~mol}$ ) was added. The reaction mixture was refluxed for 6-8 hrs. The excess of the solvent was removed under reduced pressure , aqueous $\mathrm{NaHCO}_{3}$ solution was added and the obtained semisolid was extracted with ether. The ether layer was washed thoroughly with water, dried over anhydrous $\mathrm{Na}_{2} \mathrm{SO}_{4}$, evaporate and the separated solid was collected as yellow crystal; yield: $33 \%$; mp $115-118^{\circ} \mathrm{C} ; \mathrm{IR}\left(\lambda\right.$ max, $\left.\mathrm{cm}^{-1}\right)$ : 3383 , 3279(2NH), 3173( $\left.\mathrm{CH}_{\text {arom. }}\right), 2924\left(\mathrm{CH}_{\text {aliphatic }}\right), 1617(\mathrm{C}=\mathrm{N}), 1461(\mathrm{C}=\mathrm{C}), 1083(\mathrm{C}-\mathrm{S}) ;{ }^{1} \mathrm{H}-\mathrm{NMR}\left(\mathrm{DMSO}-\mathrm{d}_{6}\right), \delta \mathrm{ppm}: 7.91-7.06(\mathrm{~m}, 10 \mathrm{H}, \mathrm{CH}$ arom.), 6.77(s,1H,NH, disappeared by $\left.\mathrm{D}_{2} \mathrm{O}\right), 6.48\left(\mathrm{~s}, 1 \mathrm{H}, \mathrm{CH}_{\text {oliph.. }}\right), 5.45\left(\mathrm{~s}, 1 \mathrm{H}, \mathrm{NH}\right.$, disappeared by $\left.\mathrm{D}_{2} \mathrm{O}\right), 3.04-3.03\left(\mathrm{~d}, 2 \mathrm{H}, \mathrm{CH}_{2}\right), 2.86-2.82(\mathrm{t}, 1 \mathrm{H}, \mathrm{CH}-$ ph); MS: (m/z,I \%): 303 (3.1) (M+1), 243 (2.6), $226(3.5), 165(7.0), 126(14.4), 107$ (17.0), 84(100), 76(2.6), calc. for $\mathrm{C}_{19} \mathrm{H}_{16} \mathrm{~N}_{2} \mathrm{~S}(\mathrm{M} . \mathrm{wt} .=304.4)$; 


\section{Synthesis of 4,6-diphenyl-4,5-dihydro-1H-indazole (7).}

To a solution of compound $6(2.95 \mathrm{~g}, 0.01 \mathrm{~mol})$ in ethanol $(20 \mathrm{ml})$, hydrazine hydrate $(0.31 \mathrm{ml}, 0.015 \mathrm{~mol})$ and a catalytic amount of triethylamine were added. The reaction mixture was refluxed for $6 \mathrm{hrs}$. The excess of solvent was evaporated under reduced pressure, the obtained solid was collected, dried and crystallized from ethanol as yellow crystal; yield: 94\%; mp 154-157우; IR $\left(\lambda \max , \mathrm{cm}^{-1}\right): 3356,3209(\mathrm{NH}), 3037\left(\mathrm{CH}_{\text {arom. }}\right)$, 2904( $\mathrm{CH}_{\text {aliphatic }}$ ), 1649(C=N), 1562 (C=C), 1442(C-N); ${ }^{1} \mathrm{H}-\mathrm{NMR}$ (DMSO-d6), $\delta \mathrm{ppm}: 7.51-7.35\left(\mathrm{~m}, 10 \mathrm{H}, \mathrm{CH}_{\text {arom }}\right) ; 6.58\left(\mathrm{~s}, 1 \mathrm{H}, \mathrm{CH}_{\text {oliph. }}\right) ; 6.35(\mathrm{~s}, 1 \mathrm{H}, \mathrm{NH}$, disappear by $\mathrm{D}_{2} \mathrm{O}$ ); 3.08-3.06(m,1H, CH-ph ); 2.85-2.84 (d,1H,CH=N); 2.81-2.80 (d, 1H, =CH-CHPh); $2.73-2.72(\mathrm{~d}, 1 \mathrm{H},=\mathrm{CH}-\mathrm{C}-\mathrm{ph}) ;{ }^{13} \mathrm{C}-\mathrm{NMR}(\mathrm{DMSO}-$ $\left.\mathrm{d}_{6}\right), \quad \delta \quad$ ppm:29.76 $\left(\mathrm{CH}_{2}\right), \quad 34.82(\mathrm{CH}-\mathrm{ph}), 125.2\left(\mathrm{CH}_{\text {oliph. }}\right), 125.3(\mathrm{CH}=\mathrm{N}), 126.87,127.64,128.60,128.9,137.5,140.3(\mathrm{C}$ of $\mathrm{Ph} \quad$ ring $), 145.8 \quad(\mathrm{CH}=\mathrm{C}-$ ph),146.1(=CH-N); MS: (m/z, I \%):269(22.7)(M+3), 210(31.8), 185(31.8), 160(36.4), 145(36.4), 121(59.1), 105 (54.5) , 91(50.0), 64(63.6). calc. for $\mathrm{C}_{19} \mathrm{H}_{16} \mathrm{~N}_{2}$ (M. wt. $\left.=272.3\right)$.

\section{Synthesis of compounds (8) and (9).}

To a solution of compound $7(2.95 \mathrm{~g}, 0.01 \mathrm{~mol})$ in ethanol $(20 \mathrm{ml})$, the proper amine (ethylenediamine or cystamine hydrochloride) $(0.01 \mathrm{~mol})$ and triethylamine $(1.4 \mathrm{ml}, 0.01 \mathrm{~mol})$ were added. The reaction mixture was refluxed for $6 \mathrm{hrs}$. The separated product was filtered off and crystallized from suitable solvent.

\section{6,8-Diphenyl-2,3,4,6-tetrahydro-1H-benzo[1,4-e] diazepine (8)}

Pale yellow crystal from ethanol; yield: 70\%; mp 197-199C; IR $\left.\left(\lambda \max , \mathrm{cm}^{-1}\right): 3415(\mathrm{NH}-), 3053 \mathrm{CH}_{\text {arom. }}\right), 2934\left(\mathrm{CH}_{\text {aliphatic }}\right), 1663(\mathrm{C}=\mathrm{N}), 1602(\mathrm{C}=\mathrm{C})$, 1474(C-N); ${ }^{1} \mathrm{H}-\mathrm{NMR}$ (DMSO-d6), $\delta \mathrm{ppm}: 7.71-7.20(\mathrm{~m}, 10 \mathrm{H}, \mathrm{CH}$ arom.), 6.73(s, $1 \mathrm{H}, \mathrm{CH}=\mathrm{N}), 6.59(\mathrm{~s}, 1 \mathrm{H}, \mathrm{CH}$ oliph.), 6.50(t, $1 \mathrm{H}, \underline{\mathrm{CH}}-\mathrm{CH}=\mathrm{N}), 5.61(\mathrm{~s}, 1 \mathrm{H}, \mathrm{NH})$, 4.67(t, 2H, CH $-\mathrm{N}), 3.69\left(\mathrm{t}, 2 \mathrm{H}, \mathrm{CH}_{2}-\mathrm{NH}\right), 3.03(\mathrm{~d}, 1 \mathrm{H}, \mathrm{CH}=\mathrm{C}-\mathrm{ph}), 2.5(\mathrm{~m}, 1 \mathrm{H}, \mathrm{CH}-\mathrm{ph})$, calc. for $\mathrm{C}_{21} \mathrm{H}_{20} \mathrm{~N}_{2}(\mathrm{M} . w \mathrm{t}=300.4)$. 


\section{6,8-Diphenyl-2,3,4,6-tetrahydro-1H-benzo[1,4-f] thiazepine (9)}

Yellow crystal for ethanol; yield: 48\%; mp>300ㄷ;IR $\left(\lambda \max , \mathrm{cm}^{-1}\right): 3437(\mathrm{NH}), 3059\left(\mathrm{CH}_{\text {arom }}\right), 2928\left(\mathrm{CH}_{\text {aliphatic }}\right), 1660(\mathrm{C}=\mathrm{N}), 1452(\mathrm{C}=\mathrm{C}), 1122(\mathrm{C}-\mathrm{S}) ;{ }^{1} \mathrm{H}-$ NMR (DMSO-d6), $\delta$ ppm : 8.18(s,1H,NH); 7.86-7.33(m, 10H, $\left.\mathrm{CH}_{\text {arom. }}\right) ; 6.92\left(\mathrm{~s}, 1 \mathrm{H}, \mathrm{CH}_{\text {oliph. }}\right), 6.79(\mathrm{~s}, 1 \mathrm{H}, \mathrm{CH}-\mathrm{NH}), 3.11(\mathrm{t}, 2 \mathrm{H}, \mathrm{CH}-\mathrm{S}), 3.02(\mathrm{~d}, 1 \mathrm{H}, \underline{\mathrm{CH}}=\mathrm{C}-$ $\mathrm{Ph}), 2.88(\mathrm{~d}, 1 \mathrm{H}, \mathrm{CH}-\mathrm{Ph}), 2.74\left(\mathrm{t}, 2 \mathrm{H}, \mathrm{CH}_{2}-\mathrm{NH}\right)$, calc. for $\mathrm{C}_{21} \mathrm{H}_{19} \mathrm{NS}(\mathrm{M} . \mathrm{wt} .=317.44)$.

\section{Synthesis of ethyl 4,6-diphenyl-4,5-dihydro-1-benzo[b]thiophen-2-yl-2-carboxylate (10).}

A mixture of compound $7(2.95 \mathrm{~g}, 0.01 \mathrm{~mol})$, ethyl mercaptoacetate $(1.096 \mathrm{ml}, 0.01 \mathrm{~mol})$ and sodium ethoxide solution $(0.23 \mathrm{~g}$ of Na dissolved in $20 \mathrm{ml}$ of ethanol) was refluxed for $6 \mathrm{hrs}$. The mixture was concentrated, the formed precipitate was filtered off and crystallized from ethanol as yellow crystal; yield: 47\%; mp169-170 ${ }^{\circ} \mathrm{C} ; \mathrm{IR}\left(\lambda \max , \mathrm{cm}^{-1}\right)$ : 3052 $\left(\mathrm{CH}_{\text {arom }}\right), 2923\left(\mathrm{CH}_{\text {aliphatic }}\right), 1661\left(\mathrm{C}=\mathrm{O}_{\text {ester }}\right), 1450(\mathrm{C}=\mathrm{C}) ;{ }^{1} \mathrm{H}-\mathrm{NMR}(\mathrm{DMSO}-\mathrm{d} 6), \delta$ ppm: 7.80-7.10(m, 10H, $\left.\mathrm{CH}_{\text {arom. }}\right) ; 6.92\left(\mathrm{~s}, 1 \mathrm{H}, \mathrm{CH}\right.$ oliph.), 6.87(d, 1H, CH=C-COOEt) , 4.10-4.03(q,2H, $\left.\underline{\mathrm{CH}_{2}}-\mathrm{CH}_{3}\right), 3.61(\mathrm{t}, 1 \mathrm{H}, \underline{\mathrm{CH}}-\mathrm{CH}$ ph), 3.02(d,1H, $\underline{\mathrm{CH}}=\mathrm{C}-\mathrm{ph}), 2.31-2.29(\mathrm{t}, 1 \mathrm{H}, \mathrm{CH}-\mathrm{Ph}), 1.22\left(\mathrm{t}, 3 \mathrm{H}, \mathrm{CH}_{3}\right)$, calc. for $\mathrm{C}_{23} \mathrm{H}_{20} \mathrm{O}_{2} \mathrm{~S}(\mathrm{M} . \mathrm{wt} .=360.4)$.

\section{Synthesis of 2E-(3,5-diphenylcyclohex-2-en-1-ylidene)hydrazene- $N^{\prime}[(2 R, 3 R, 4 R, 5 R)-2,3,4,5,6$-penta- hydroxyhexylidene(12).}

To a solution of compound $11(2.62 \mathrm{~g}, 0.01 \mathrm{~mol})$ in dimethylformamide $(20 \mathrm{ml}), D(+)$ glucose $(1.80 \mathrm{~g}, 0.01 \mathrm{~mol})$ and $(0.1 \mathrm{ml})$ acetic acid were added. The mixture was heated under reflux on a water-bath for 2 hrs. The separated solid on cooling was filtered off and crystallized from ethanol as pale brown crystal; yield: $85 \%$; mp 220-222 ${ }^{\circ} \mathrm{C}$; IR $\left(\lambda \max , \mathrm{cm}^{-1}\right): 3433(\mathrm{OH}$ and $\mathrm{NH}), 3045\left(\mathrm{CH}_{\text {arom. }}\right), 2924\left(\mathrm{CH}_{\text {aliphatic }}\right), 1637(\mathrm{C}=\mathrm{N}), 1605$ $(\mathrm{C}=\mathrm{C}), 1438(\mathrm{C}-\mathrm{N}) ;{ }^{1} \mathrm{H}-\mathrm{NMR}\left(\mathrm{CDCl}_{3}\right), \delta \mathrm{ppm}: 7.89-7.16(\mathrm{~m}, 10 \mathrm{H}, \mathrm{CH}$ arom $), 7.45\left(\mathrm{~s}, 5 \mathrm{H}, 5 \mathrm{OH}\right.$, disappeared by $\left.\mathrm{D}_{2} \mathrm{O}\right), 6.56(\mathrm{~s}, 1 \mathrm{H}, \mathrm{CH}$ oliph.), 3.083.07(d, 1H, CH=N), 2.99-2.97(d, 2H, $\left.\underline{\mathrm{CH}}_{2}-\mathrm{CHPh}\right), 2.90-2.92(\mathrm{t}, 1 \mathrm{H}, \mathrm{CH}-\mathrm{OH}), 2.84-2.80\left(\mathrm{~d}, 2 \mathrm{H}, \mathrm{CH}_{2}-\mathrm{OH}\right), 3.01-2.79\left(\mathrm{~d}, 2 \mathrm{H}, \mathrm{CH}_{2}\right), 2.79-2.71(\mathrm{q}$, $3 \mathrm{H}, \mathrm{CH}-\mathrm{OH}), 2.49(\mathrm{~m}, 1 \mathrm{H}, \mathrm{CH}-\mathrm{Ph}) ;{ }^{13} \mathrm{C}-\mathrm{NMR}\left(\mathrm{CDCl}_{3}, \delta \mathrm{ppm}: 32.49,32.53,35.82\left(\mathrm{CH}_{2}-\mathrm{OH}+\mathrm{CH}_{2}-\mathrm{C}=\mathrm{N}+\mathrm{CH}_{2}-\mathrm{C}-\mathrm{Ph}\right) ; 40.62,40.25,40.11,36.01\right.$, 
(4CH-OH); 116.21(ㅌH-Ph); 124.65(CH oliph.), 125.56,126.09,126.78,127.06,128.57,128.67 (C of Ph. ring); 139.76(=C-Ph); 144.63,147.37 (2 $\mathrm{C}=\mathrm{N}$ ), calc. for $\mathrm{C}_{24} \mathrm{H}_{28} \mathrm{~N}_{2} \mathrm{O}_{5}$ (M. wt. $=424.4$ ).

\section{Synthesis of (3,5-diphenylcyclohex-2-en-1-ylidene)hydrazene- $N^{\prime}$-(4-nitrobenzylidene) (13).}

A mixture of compound $5(2.62 \mathrm{~g}, 0.01 \mathrm{~mol})$ and p-nitrobenzaldhyde $(1.51 \mathrm{~g}, 0.01 \mathrm{~mol})$ in glacial acetic acid (20 ml) was refluxed with stirring for $5 \mathrm{hrs}$. After cooling, the formed solid was filtered off and recrystallized from ethanol as pale yellow crystal; Yield: $62 \%$; $\mathrm{mp} 156-158^{\circ} \mathrm{C}$; IR $(\lambda \mathrm{max}$, $\left.\mathrm{cm}^{-1}\right): 3443(\mathrm{NH}), 3047\left(\mathrm{CH}_{\text {arom }}\right), 2927\left(\mathrm{CH}_{\text {aliphatic }}\right), 1610(\mathrm{C}=\mathrm{N}), 1597(\mathrm{C}=\mathrm{C}), 1518-1339\left(\mathrm{NO}_{2}\right) ;{ }^{1} \mathrm{H}-\mathrm{NMR}\left(\mathrm{DMSO}-\mathrm{d}_{6}\right), \delta \mathrm{ppm}: 8.85(\mathrm{~s}, 1 \mathrm{H}, \mathrm{NH}), 8.58-$ 8.06(m,4H,Ar-NO $\left.)_{2}\right), 7.70-7.26\left(\mathrm{~m}, 10 \mathrm{H}, \mathrm{CH}_{\text {arom. }}\right), 7.50(\mathrm{~s}, 1 \mathrm{H}, \mathrm{CH}=\mathrm{N}), 6.86\left(\mathrm{~s}, 1 \mathrm{H}, \mathrm{CH}_{\text {oliph. }}\right), 3.51,3.45(\mathrm{~d}, 1 \mathrm{H}, \mathrm{CH}), 2.96-2.93(\mathrm{~d}, 2 \mathrm{H}, \mathrm{CH}), 2.74-2.68(\mathrm{~m}$, $1 \mathrm{H}, \mathrm{CH}-\mathrm{Ph}$ ), calc. for $\mathrm{C}_{25} \mathrm{H}_{21} \mathrm{~N}_{3} \mathrm{O}_{2}$ (M. wt.= 395).

\section{Synthesis of 3-(3,5-diphenylcyclohexa-1,5-dienylamino)-2-(4-nitrophenyl)-1,3-thiazolidin-4-one (14).}

Thioglycolic acid $(0.69 \mathrm{ml}, 0.01 \mathrm{~mol})$ was added drop by drop to a solution of compound $13(3.95 \mathrm{~g}, 0.01 \mathrm{~mol})$ in dry benzene $(30 \mathrm{ml})$. The reaction mixture was refluxed for $10 \mathrm{hrs}$ and the solvent evaporated under reduced pressure. The solid was triturated with petroleum ether $\left(60-80{ }^{\circ} \mathrm{C}\right)$, filtered off, dried and crystallized from $\mathrm{CHCl}_{3} /$ pet.ether $\left(40-60{ }^{\circ} \mathrm{C}\right)$ as deep yellow crystal; yield: $52 \%$; mp $216-218^{\circ} \mathrm{C}$; IR $\left(\lambda\right.$ max, $\left.\mathrm{cm}^{-1}\right): 3403$ (NH),3084 ( $\left.\mathrm{CH}_{\text {arom }}\right), 2929\left(\mathrm{CH}_{\text {aliph }}\right), 1718(\mathrm{C}=\mathrm{O}), 1595(\mathrm{C}-\mathrm{N}), 1517-1337\left(\mathrm{NO}_{2}\right), 1111(\mathrm{C}-\mathrm{S})$; ${ }^{1} \mathrm{H}-\mathrm{NMR}\left(\mathrm{DMSO}-\mathrm{d}_{6}\right), \delta$ ppm: 8.84-8.15(m,4H,CHof ph- $\left.\mathrm{NO}_{2}\right), 7.64-7.24\left(\mathrm{~m}, 10 \mathrm{H}, \mathrm{CH}_{\text {arom. }}\right), 6.87\left(\mathrm{~s}, 1 \mathrm{H}, \mathrm{CH}-\mathrm{Ph}-\mathrm{NO}_{2}\right), 6.80\left(\mathrm{~s}, 1 \mathrm{H}, \mathrm{CH}_{\text {oliph. }}\right), 4.11\left(\mathrm{~s}, 2 \mathrm{H}, \mathrm{CH}_{2}-\mathrm{C}=\mathrm{O}\right), 3.65,3.64(\mathrm{~d}, 1 \mathrm{H}, \underline{\mathrm{CH}}=\mathrm{C}), 2.89(\mathrm{~d}, 2 \mathrm{H}$, $\left.\mathrm{CH}_{2}\right), 2.73(\mathrm{~m}, 1 \mathrm{H}, \mathrm{CH}-\mathrm{ph}), 2.50(\mathrm{~s}, 1 \mathrm{H}, \mathrm{NH})$, calc. for $\mathrm{C}_{27} \mathrm{H}_{23} \mathrm{~N}_{3} \mathrm{O}_{3} \mathrm{~S}(\mathrm{M} . \mathrm{wt} .=469.55)$. 


\section{Synthesis of 2-(3,5-diphenylcyclohex-2-enylidene)-N-phenylhydrazinecarbothioamide (15).}

A solution of compound $11(2.62 \mathrm{~g}, 0.01 \mathrm{~mol})$ and phenylisothiocyanate $(0.135 \mathrm{~g}, 0.01 \mathrm{~mol})$ in dry dioxane (30 ml) was refluxed for $5 \mathrm{hrs}$. The reaction mixture was concentrated under reduced pressure, the obtained solid was filtered off and recrystallized from ethanol. Yellow crystal; yield: 47\%; (mp. Literature $\left.{ }^{24}\right)$; IR $\left(\lambda \max , \mathrm{cm}^{-1}\right): 3424(2 \mathrm{NH}), 3036\left(\mathrm{CH}_{\text {arom }}\right), 2927\left(\mathrm{CH}_{\text {aliph. }}\right), 1606(\mathrm{C}=\mathrm{N}) ;{ }^{1} \mathrm{H}$ NMR $\left(400 \mathrm{MHz}, \mathrm{DMSO}-\mathrm{d}_{6}\right) \delta 7.26$ (s, 1H, NH), 7.24(s, 1H, NH); 7.64-7.37(m, 15H, $\left.\mathrm{CH}_{\text {arom. }}\right), 6.87(\mathrm{~s}, 1 \mathrm{H}, \mathrm{SH}), 6.79\left(\mathrm{~s}, 1 \mathrm{H}, \mathrm{CH}_{\text {oliph }}\right), 3.56(\mathrm{~d}, 1 \mathrm{H}, \mathrm{CH}=\mathrm{C}-\mathrm{NH}), 3.18(\mathrm{~m}, 1 \mathrm{H}, \mathrm{CH}-\mathrm{ph})$;

$2.87\left(\mathrm{~d}, 2 \mathrm{H}, \mathrm{CH}_{2}\right) ;{ }^{13} \mathrm{C}$ NMR(400 MHz,DMSO-d $\left.{ }_{6}\right) \delta$ 161.51(C-SH), 147.20(N=C-NPh), 144.67(C-NH), 125.55, 126.77, 127.06,128.57, 128.67, $139.80(\mathrm{C}$ of $\mathrm{Ph}), 124.74\left(\mathrm{CH}_{\text {oliph }}\right), 67.10(\underline{\mathrm{CH}}=\mathrm{C}-\mathrm{NH}), 40.11\left(\mathrm{CH}_{2}\right), 35.81(\mathrm{CH}-\mathrm{Ph})$, calc. for $\mathrm{C}_{25} \mathrm{H}_{23} \mathrm{~N}_{3} \mathrm{~S}(\mathrm{M}$. wt. $=397.5)$.

\section{Synthesis of compounds (16) and (17).}

A mixture of compound 11 (3.97 g, $0.01 \mathrm{~mol})$ and a proper dihalocompounds (chloroacetylchloride or malonyldichloride) (0.01 mol) was refluxed in dry DMF $(20 \mathrm{ml})$ for $4 \mathrm{hrs}$. After cooling, the formed solid was filtered off, and recrystallized from the proper solvent.

\section{3-(3,5-Diphenylcyclohexa-1,5-dienylamino)-2-phenyl iminothiazolidin-4-one (16).}

Orange crystal from dioxane; yield: 55\%; $\mathrm{mp} 244-246$ - $\mathrm{C} ; \mathrm{IR}\left(\lambda \max , \mathrm{cm}^{-1}\right): 3359(\mathrm{OH}), 3155(\mathrm{NH}), 3075(\mathrm{CH}$ arom. $), 2929\left(\mathrm{CH}_{\text {aliphatic. }}\right), \quad 1612(\mathrm{C}=\mathrm{N})$, 1460(C=C), 1407 (C-N); ${ }^{1} \mathrm{H}-\mathrm{NMR}\left(\mathrm{DMSO}-\mathrm{d}_{6}\right), \delta \mathrm{ppm}:$ 9.03-8.95(s, 2H, OH and NH),7.95-7.27 (m,14H, $\left.\mathrm{CH}_{\text {arom. }}\right), 7.11(\mathrm{~s}, 1 \mathrm{H}, \mathrm{CH}$ oliph), 4.23(s, 1H, CH-S), 3.76(d, $1 \mathrm{H}, \mathrm{CH}=\mathrm{C}), 3.06(\mathrm{~m}, 1 \mathrm{H}, \mathrm{CH}-\mathrm{ph}), 2.88-2.72\left(\mathrm{~d}, 2 \mathrm{H}, \mathrm{CH}_{2}\right) ;{ }^{13} \mathrm{C}-\mathrm{NMR}$ (DMSO-d $\left.\mathrm{d}_{6}\right), \delta \mathrm{ppm}: 36.55\left(\mathrm{CH}_{2}\right), 41.05(\mathrm{CH}-\mathrm{Ph}), 43.97(\mathrm{CH}-\mathrm{S}), 67.09$ $(\mathrm{CH}=\mathrm{C}), 125.15\left(\mathrm{CH}_{\text {oliph }}\right), 125.56,126.22,126.83,127.05,127.14,127.60,128.69,128.87,129.87,130.23$ (C of Ph ring), 138.38(C- $\left.\mathrm{Ph}\right), 143.22(\underline{\mathrm{C}}-\mathrm{NH})$,

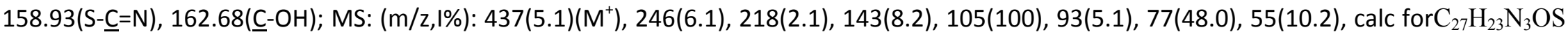
(M. wt.=437.5). 
3-(3,5-Diphenyl-cyclohexa-1,5-dienylamino)-6-hydroxy-2-phenylimino-2,3-dihydro-[1,3] thiazine-4-one (17).

Brown powder from DMF; yield: 43\%; mp 126-128ㄷ; IR $\left(\lambda \max , \mathrm{cm}^{-1}\right)$ : $3407(\mathrm{OH}), 3042\left(\mathrm{CH}_{\text {arom. }}\right), 2938(\mathrm{CH}$ aliphatic $), 1733(\mathrm{C}=\mathrm{O}), 1657(\mathrm{C}=\mathrm{N})$, 1493(C=C),1435 (C-N); ${ }^{1} \mathrm{H}-\mathrm{NMR}$ (DMSO - $\left.\mathrm{d}_{6}\right), \delta \mathrm{ppm}$ : 7.72-7.28(m,14H,CHarom.), 6.48(s,1H,CH oliph), $4.25(\mathrm{~s}, 1 \mathrm{H}, \mathrm{CH}=\mathrm{C}-\mathrm{OH}) ; 4.10(\mathrm{~d}, 1 \mathrm{H}, \mathrm{CH}=\mathrm{C}-\mathrm{NH})$, 3.58(s, $1 \mathrm{H}, \mathrm{NH}$, disappeared by $\left.\mathrm{D}_{2} \mathrm{O}\right), 3.45(\mathrm{~m}, 1 \mathrm{H}, \mathrm{CH}-\mathrm{Ph}), 3.03\left(\mathrm{~d}, 2 \mathrm{H}, \mathrm{CH}_{2}\right), 2.82\left(\mathrm{~s}, 1 \mathrm{H}, \mathrm{OH}\right.$, disappeared by $\left.\mathrm{D}_{2} \mathrm{O}\right) .{ }^{13} \mathrm{C}-\mathrm{NMR}\left(\mathrm{DMSO}-\mathrm{d}_{6}\right), \delta$ ppm: 37.23( $\left(\mathrm{CH}_{2}\right), 42.18(\mathrm{CH}-\mathrm{ph}), 59.17(\mathrm{CH}=\mathrm{C}), 64.05(\underline{\mathrm{CH}}=\mathrm{C}-\mathrm{OH}), 124.67\left(\mathrm{CH}_{\text {oliph. }}\right), 92.01,126.83,127.13,127.54,128.97,129,129.27(\mathrm{C}$ of Ph ring.), 130.26 (=C-Ph), 138.51(C=N), 144.21(C-NH), 159.10(C-OH), 198.80(C=O), calc. for $\mathrm{C}_{28} \mathrm{H}_{23} \mathrm{~N}_{3} \mathrm{O}_{2} \mathrm{~S}$ (M. wt. $\left.=465.3\right)$.

\section{Synthesis of ethyl [(2E)-2-(3,5-diphenylcyclohex-2-en-1-ylidene)hydrazine]acetate (18)}

To a solution of compound $\mathbf{1 1}(2.62 \mathrm{~g}, 0.01 \mathrm{~mol})$ in dioxane $(20 \mathrm{ml})$, ethyl bromoacetate $(1.10 \mathrm{ml}, 0.01 \mathrm{~mol})$ and triethylamine $(1.4 \mathrm{ml}, 0.01 \mathrm{~mol})$ were added .The reaction mixture was refluxed for $6 \mathrm{hrs}$. After cooling, the separated solid was collected and crystallized from ethanol as yellow crystal; yield:64\%; mp 250-252우 $/ \mathrm{IR}\left(\lambda \max , \mathrm{cm}^{-1}\right): 3361,3151(2 \mathrm{NH}), 3037\left(\mathrm{CH}_{\text {arom }}\right), 2927\left(\mathrm{CH}_{\text {aliphatic }}\right), 1740(\mathrm{C}=\mathrm{O}) 1598(\mathrm{C}=\mathrm{C}) ;{ }^{1} \mathrm{H}-\mathrm{NMR}\left(\mathrm{CDCl}{ }_{3}\right), \delta$ ppm: 7.61-6.90(m, 10H, $\left.\mathrm{CH}_{\text {arom. }}\right), 6.79\left(\mathrm{~s}, 1 \mathrm{H}, \mathrm{CH}_{\text {oliph }}\right), 4.15,3.63\left(\mathrm{~s}, 2 \mathrm{H}, 2 \mathrm{NH}\right.$, disappeared by $\left.\mathrm{D}_{2} \mathrm{O}\right), 3.39-3.35(\mathrm{~m}, 1 \mathrm{H}, \underline{\mathrm{CH}}-\mathrm{NH}), 3.15-3.09(\mathrm{q}, 2 \mathrm{H}$, $\left.\left.\underline{\mathrm{CH}_{2}} \mathrm{CH}_{3}\right), 2.91-2.87\left(\mathrm{~d}, 2 \mathrm{H},=\mathrm{C}-\underline{\mathrm{CH}}_{2}\right), 2.79-2.72\left(\mathrm{~m}, 2 \mathrm{H}, \mathrm{NHCH} \underline{C H}_{2}\right), 2.42-2.32(\mathrm{~m}, 1 \mathrm{H}, \underline{\mathrm{CH}}-\mathrm{Ph}), 1.61\left(\mathrm{~s}, 2 \mathrm{H}, \mathrm{CH}_{2}-\mathrm{C}=\mathrm{O}\right), 1.38-1.35 \quad\left(\mathrm{t}, 3 \mathrm{H}, \mathrm{CH}_{3}\right) ;{ }^{13} \mathrm{C}-\mathrm{NMR}(\mathrm{CDCl})_{3}\right), \delta$ ppm: 31.50( $\left.\mathrm{CH}_{3}\right), 32.5\left(\mathrm{CH}_{2}\right), 36.36\left(\mathrm{CH}_{2}-\mathrm{CHPh}\right), 41.05\left(\mathrm{CH}_{2} \mathrm{C}=\mathrm{O}\right), 43.97\left(\mathrm{O}-\mathrm{CH}_{2}\right), 67.09(\mathrm{CH}-\mathrm{Ph}), 125.15(=\mathrm{CH}), 126.22(\mathrm{CH}-\mathrm{NH}), 126.83,127.14,128.69$, 128.87, 130.23,138.38,158.93,143.22 (C of Ph rings), 162.68(=C-Ph),199.39(C=O); MS: (m/z, l\%): $351(23.8)\left(M^{+}\right), 351.7(28)\left(M^{+}+1\right), 288(28.6)$, 247(71.4), 221(28.6),206(33.3),141(52.4), 128(28.6), 102(47), 91(81.0), 63(81.0), 57(100), calc.for $\mathrm{C}_{22} \mathrm{H}_{26} \mathrm{~N}_{2} \mathrm{O}_{2}$ (M. wt. = 350.45).

\section{Synthesis of 2-[(2E)-2-(3,5-diphenylcyclohex-2-en-1-ylidene)hydrazine]acetohydrazide (19).}


A mixture of compound $18(3.48 \mathrm{~g}, 0.01 \mathrm{~mol})$, hydrazine hydrate $(0.31 \mathrm{ml}, 0.01 \mathrm{~mol})$ in ethanol $(20 \mathrm{ml})$, was refluxed for 5 hrs and allowed to cool. The solid product was collected and crystallized from ethanol as yellow crystal; yield: $62 \% ; \mathrm{mp} 230-232{ }^{\circ} \mathrm{C} ; \mathrm{IR}\left(\lambda \mathrm{max}, \mathrm{cm}^{-1}\right): 3419-3357\left(\mathrm{NH}_{2}\right)$,

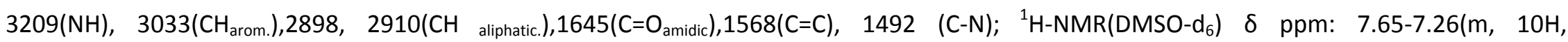
$\left.\mathrm{CH}_{\text {arom. }}\right), 6.81\left(\mathrm{~s}, 1 \mathrm{H}, \mathrm{CH}\right.$ oliph.), 5.06(s, $\left.2 \mathrm{H}, \mathrm{NH}_{2}\right), 3.68(\mathrm{~s}, 3 \mathrm{H}, 3 \mathrm{NH}), 3.57\left(\mathrm{~s}, 2 \mathrm{H}, \mathrm{CH}_{2} \mathrm{C}=\mathrm{O}\right), 3.35(\mathrm{~m}, 1 \mathrm{H}, \underline{\mathrm{CH}}-\mathrm{NH}), 3.15(\mathrm{~m}, 2 \mathrm{H}, \underline{\mathrm{CH}} \underline{2}-\mathrm{CH}-\mathrm{NH}), 2.90-2.89(\mathrm{~m}$, $1 \mathrm{H}, \mathrm{CH}-\mathrm{Ph}), 2.60\left(\mathrm{~d}, 2 \mathrm{H},=\mathrm{C}-\mathrm{CH}_{2}\right) ;{ }^{13} \mathrm{C}-\mathrm{NMR}\left(\mathrm{DMSO}-\mathrm{d}_{6}\right), \delta \mathrm{ppm}: 31.47\left(\mathrm{CH}_{2}\right), 34.97\left(\mathrm{CH}_{2}\right), 39.21\left(\mathrm{CH}_{2}-\mathrm{C}=\mathrm{O}\right), 113.63,124.18,124.53(\underline{\mathrm{C}} \mathrm{H}-\mathrm{cyclohexen}$ ring), $125.75,126,127.54,127.63,138.71$ (C of Ph ring),143.58 (=C-Ph),181.99 (C=O), calc. for $\mathrm{C}_{20} \mathrm{H}_{24} \mathrm{~N}_{4} \mathrm{O}$ (M. wt.= 336.4).

\section{Synthesis of 1-(3,5-dimethyl-1H-2,3-dihydropyrazole-1-yl)-2-[(2E)-(3,5-diphenyl-cyclohexa-1- ene)hydrazinyl) ethanone (20).}

A mixture of compound $19(3.33 \mathrm{~g}, 0.01 \mathrm{~mol})$, acetyl acetone $(0.31 \mathrm{ml}, 0.01 \mathrm{~mol})$ and a catalytic amount of triethylamine in ethanol (20 ml), was refluxed for $7 \mathrm{hrs}$. After cooling the solid product was collected and crystallized from ethanol as deep green powder; yield: 57\%; mp 262-264우 IR $\left(\lambda \max , \mathrm{cm}^{-1}\right): 3393,3185(3 \mathrm{NH}), 3064\left(\mathrm{CH}_{\text {arom. }}\right), 2929\left(\mathrm{CH}_{\text {aliphatic. }}\right), 1641(\mathrm{C}=\mathrm{O}$ amidic $), 1599(\mathrm{C}=\mathrm{N}), 1493(\mathrm{C}=\mathrm{C}), 1439(\mathrm{C}-\mathrm{N}) ;{ }^{1} \mathrm{H}-\mathrm{NMR}(\mathrm{CDCl} 3, \delta \mathrm{ppm})$ : 7.63-7.18(m,10H, $\left.\mathrm{CH}_{\text {arom. }}\right) ; 6.86\left(\mathrm{~s}, 1 \mathrm{H}, \mathrm{CH}\right.$ pyrazol ring), 6.77(s,1H, $\left.\mathrm{CH}_{\text {oliph }}\right) ; 3.63\left(\mathrm{~s}, 2 \mathrm{H}, \mathrm{CH}_{2}-\mathrm{C}=\mathrm{O}\right) ; 3.38-3.34(\mathrm{~m}, 1 \mathrm{H}, \underline{\mathrm{CH}}-\mathrm{NH}) ; 3.12(\mathrm{~s}, 2 \mathrm{H}, \mathrm{NH}$, disappeared by $\left.\mathrm{D}_{2} \mathrm{O}\right), 2.90-2.86\left(\mathrm{~m}, 2 \mathrm{H}, \mathrm{CH}_{2}\right) ; 2.78-2.75\left(\mathrm{~d}, 1 \mathrm{H},=\mathrm{C}-\mathrm{CH}_{2}\right) ; 2.43-2.31(\mathrm{~m}, 1 \mathrm{H}, \underline{\mathrm{CH}}-\mathrm{Ph}), 1.18\left(\mathrm{~s}, 6 \mathrm{H}, 2 \mathrm{CH}_{3}\right)$, calc. for ${ }_{25} \mathrm{H}_{28} \mathrm{~N}_{4} \mathrm{O}(\mathrm{M}$. wt. $=$ 400.5). 

A's -14

proton_su DMSO \{C: $\backslash \mathrm{nmr}$-data $\}$ student 20

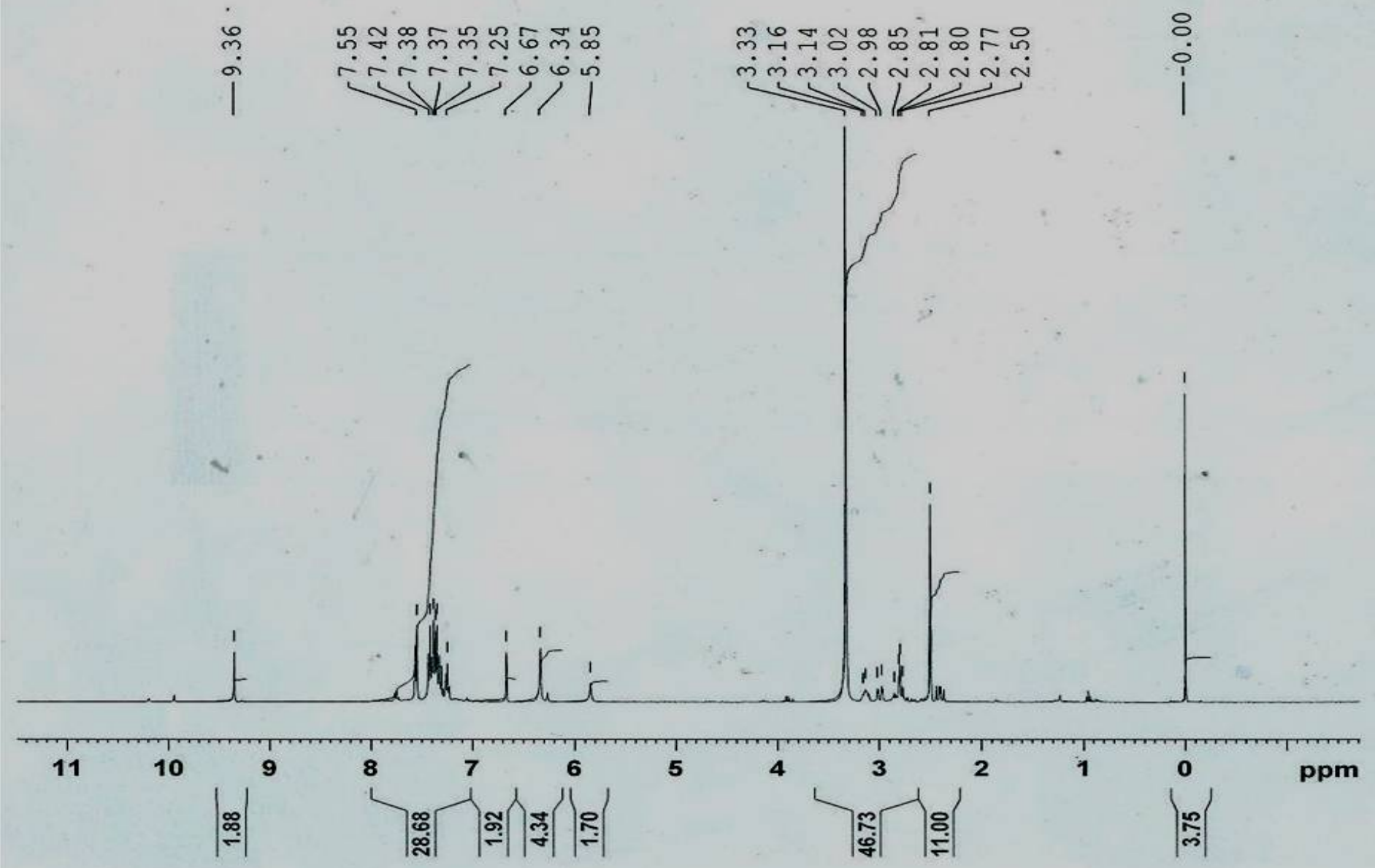

\section{हर्शिए}

$\begin{array}{lr}\text { Current Data } & \text { Parameters } \\ \text { NAME } & \text { Aug22-20113 } \\ \text { EXPNO } & 70 \\ \text { PROCNO } & 1\end{array}$

F2 - Acquisition Parameters
Date_- 20130822

Time-

INSTRUM
PROBHD

sPect
PULPROG $5 \mathrm{~mm}$ PABBO BB/

TD $\quad \begin{aligned} 2930 \\ \text { SOLVENT }\end{aligned}$

NS
DS

FWH

FIDR

$\mathrm{DW}$
$\mathrm{DE}$
$\mathrm{TE}$
$\mathrm{TE}$
$\mathrm{D} 1$

TD 1 0

SFO1
NUC1
P1

8
2
8012.820

$8012.820 \mathrm{~Hz}$
$0.122266 \mathrm{~Hz}$

199.04 sec

62.400
6.50 uses

$1.000000002 \mathrm{~K}$

400.1324710 MHz

F2 - Processing parameters

$\begin{array}{rr}\text { SI } & \\ \text { SF } & 400.1300032 \\ \text { MHz }\end{array}$

wD
SS
GB
RC
PC

$0.30 \mathrm{~Hz}$

1.00

Figure S1. ${ }^{1} \mathrm{H}$ NMR $\left(400 \mathrm{MHz}, \mathrm{DMSO}-\mathrm{d}_{6}\right)$ of compound 2. 


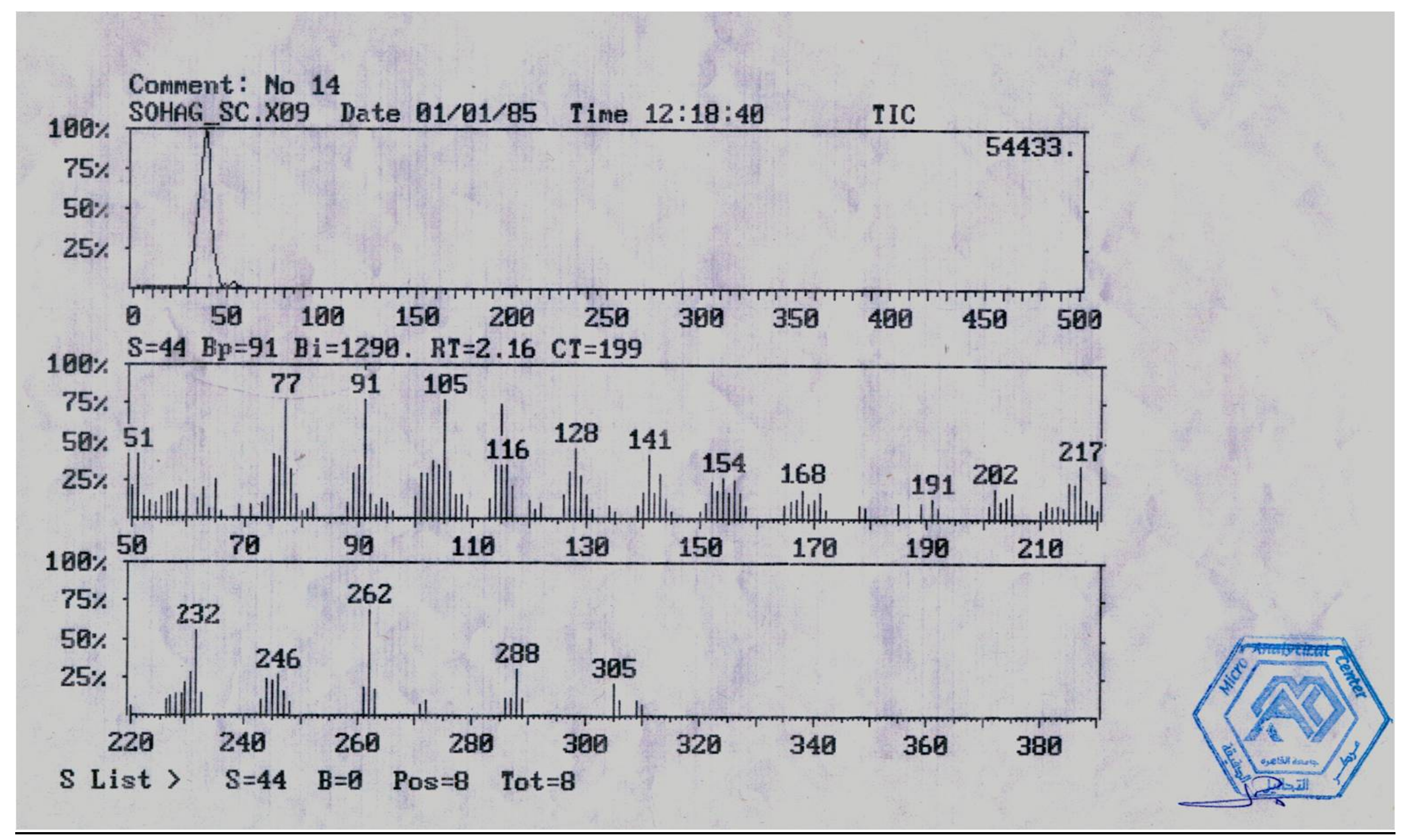

Figure S2. Mass spectra of compound 2. 



\section{Ast38}

proton_su DMSO (C: Inmr-data) Student 19

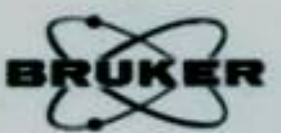

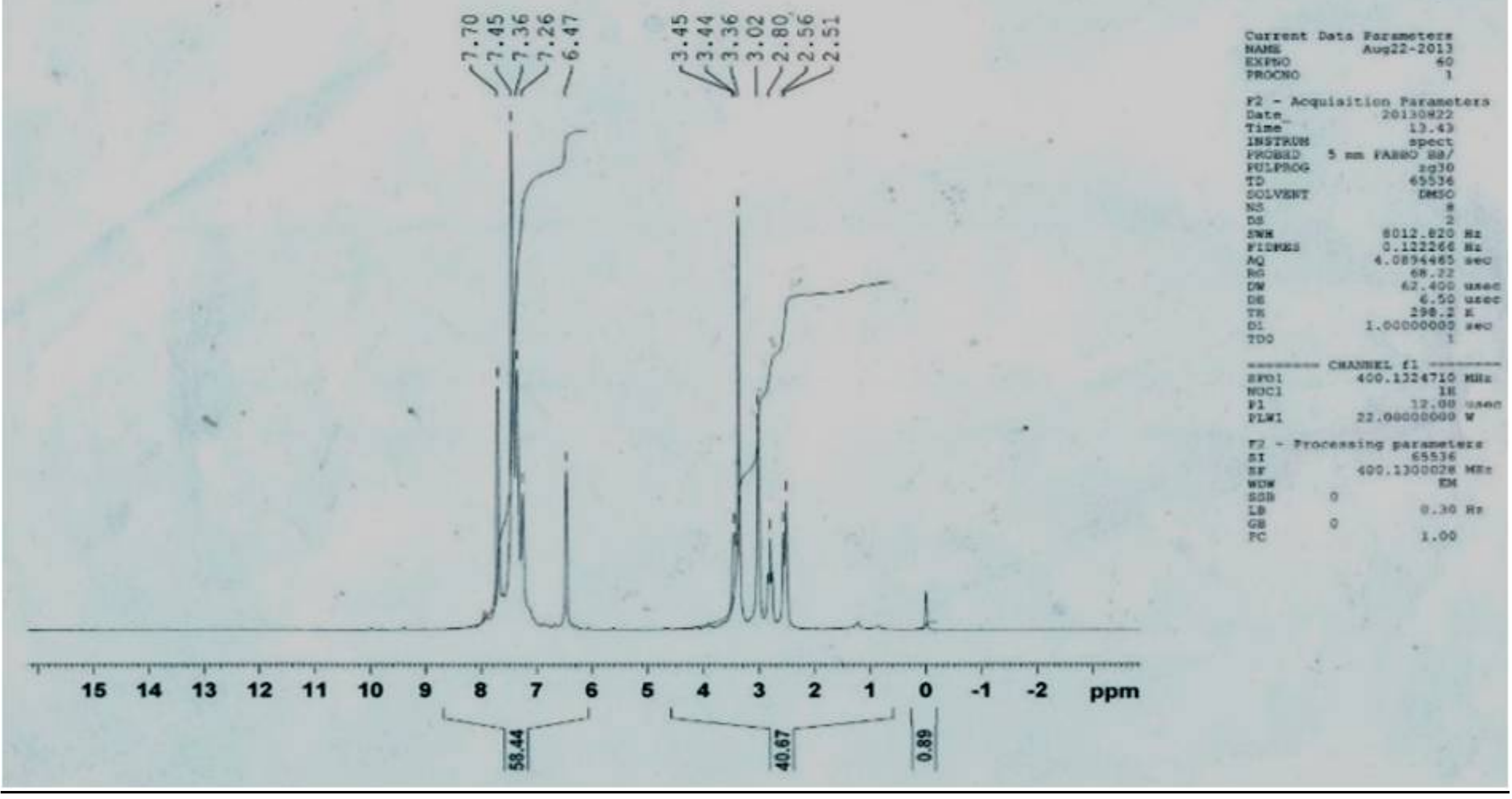

Figure S3. ${ }^{1} \mathrm{H}$ NMR $\left(400 \mathrm{MHz}\right.$, DMSO-d $\left.\mathrm{d}_{6}\right)$ of compound 3. 
Aas -33

e19_-u DMSp fC:Mnis-dacal atudent 12

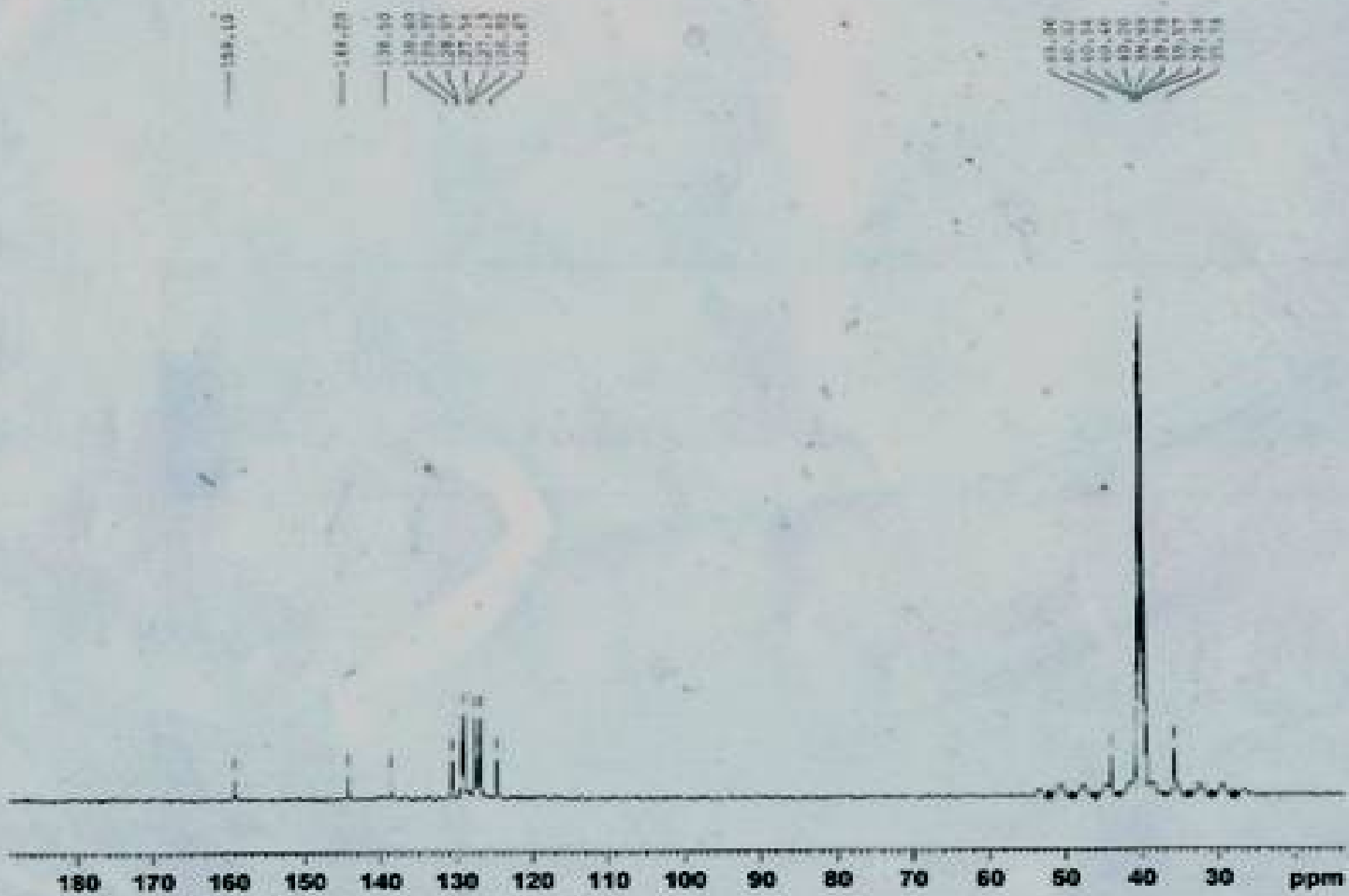

\section{afsen try}

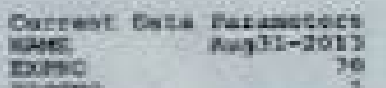

Dorct

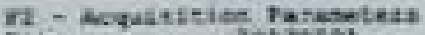

Dite- Jotristis

matrat

moan in

prestrir

47

ritson

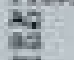

표

tis

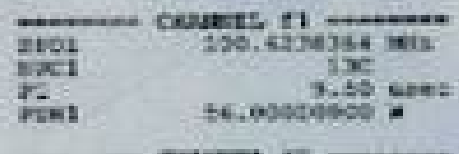

$\frac{3 n^{2}}{2}$

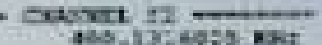

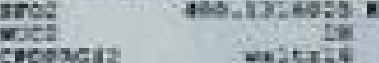
$\cot =4$

Arst

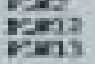

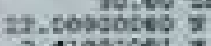

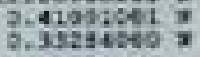

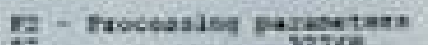

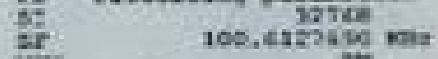

is

105.45ing kits

biet Br.

1.48

Figure S4. ${ }^{13} \mathrm{C}$ NMR (400 MHz, DMSO- $d_{6}$ ) of compound 3. 


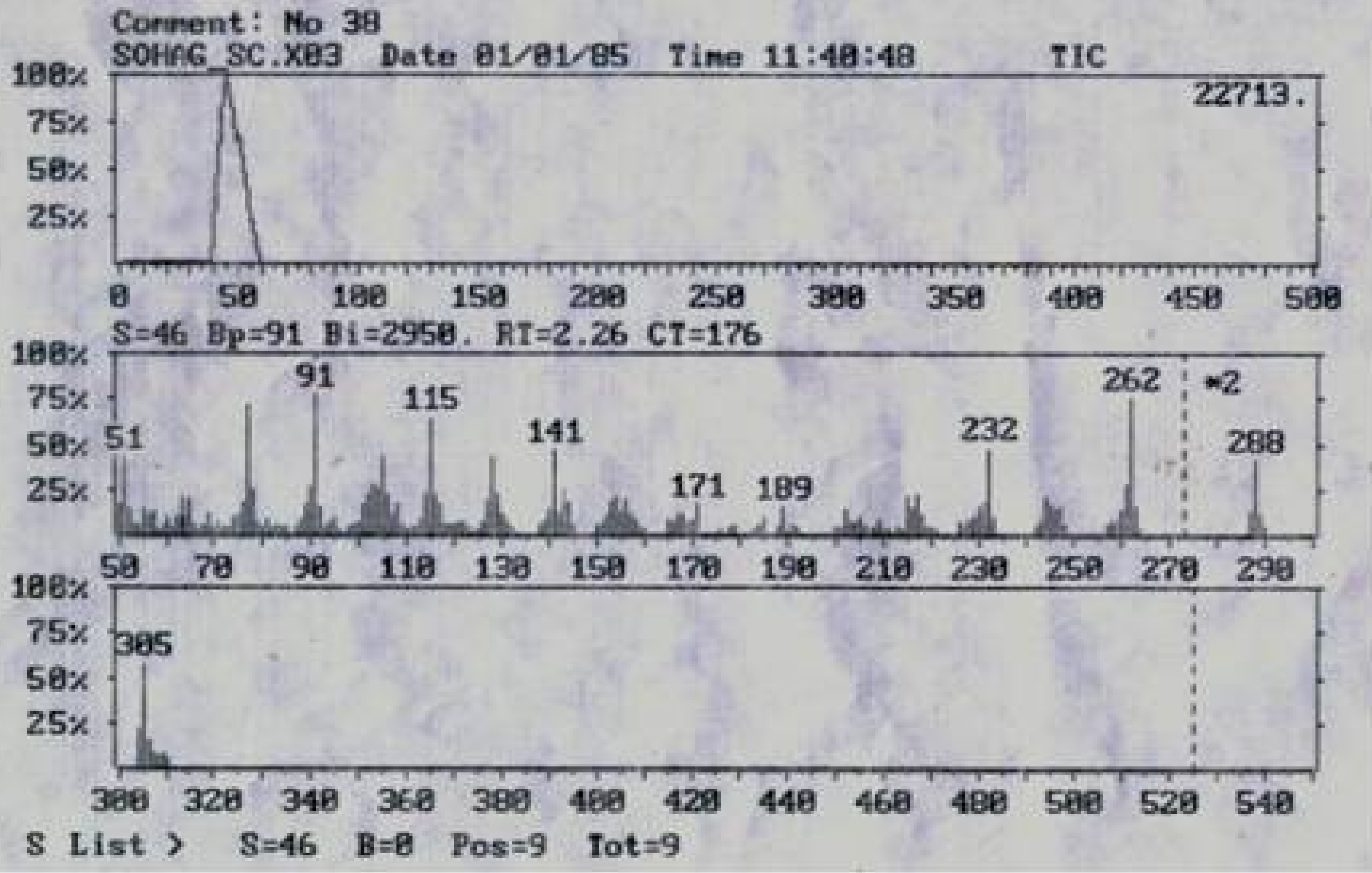

Figure S5. Mass spectra of compound 3. 
A4-1:

proton_ou muso [CqMnmr-dstal Btwdent 7
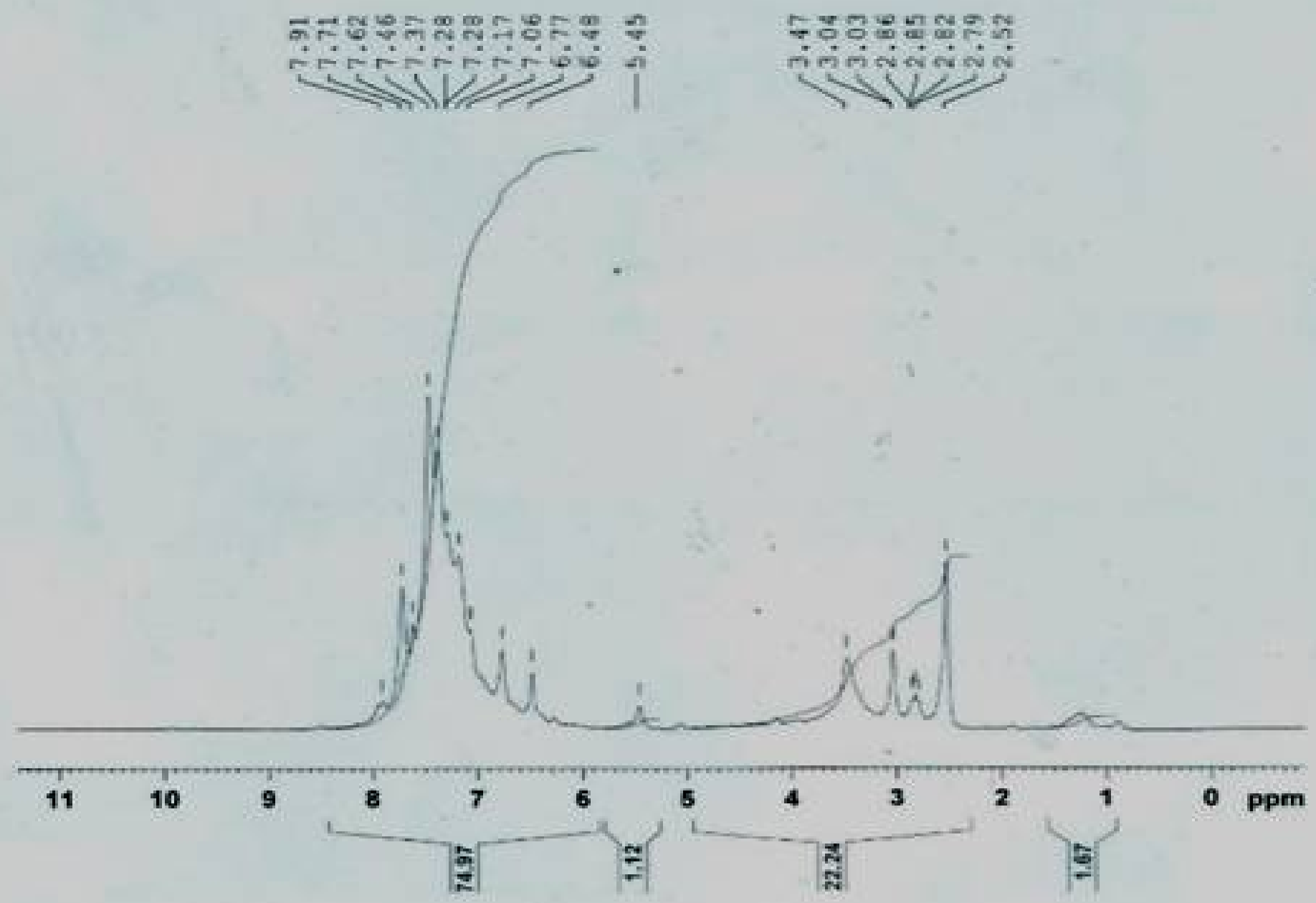

2ingers

\section{gip?ara}

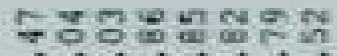

niminito

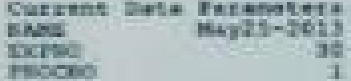

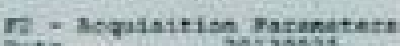

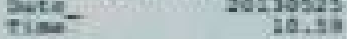

Hatres 4 - nusori

ine

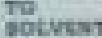

is

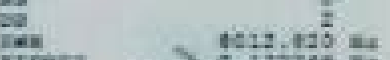

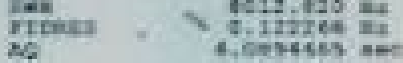

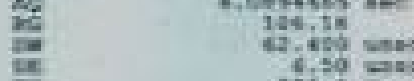

늘

柴

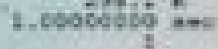

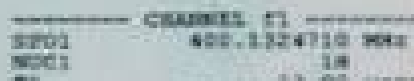

Aa.

nim anosodis =

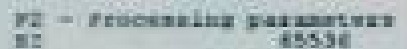

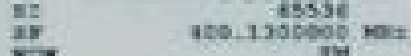

표 a in

焉 1.60 
Figure S6. ${ }^{1} \mathrm{H}$ NMR (400 MHz, DMSO-d $\mathrm{d}_{6}$ ) of compound 5. 


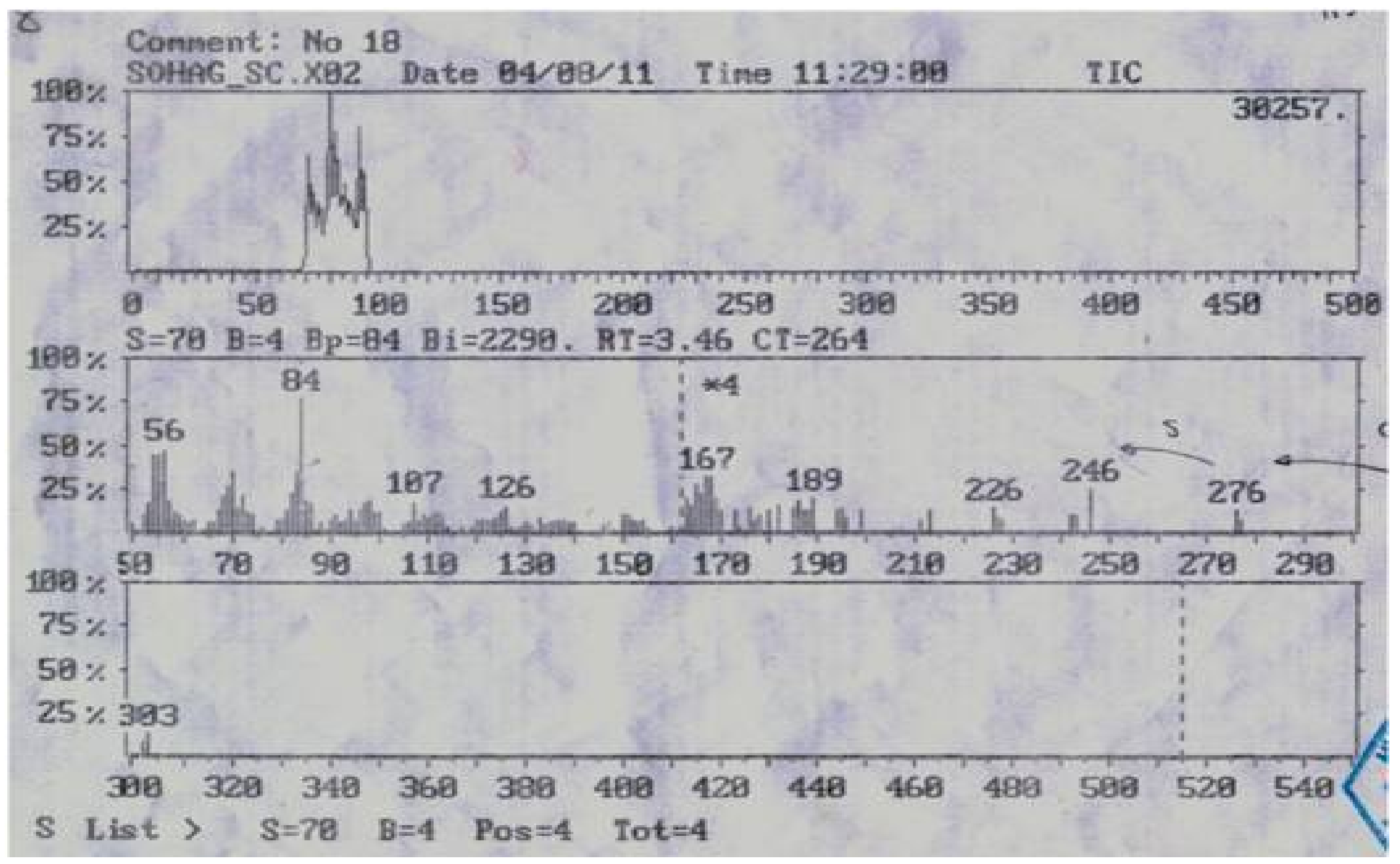


Figure S7. Mass spectra of compound $\mathbf{5}$. 


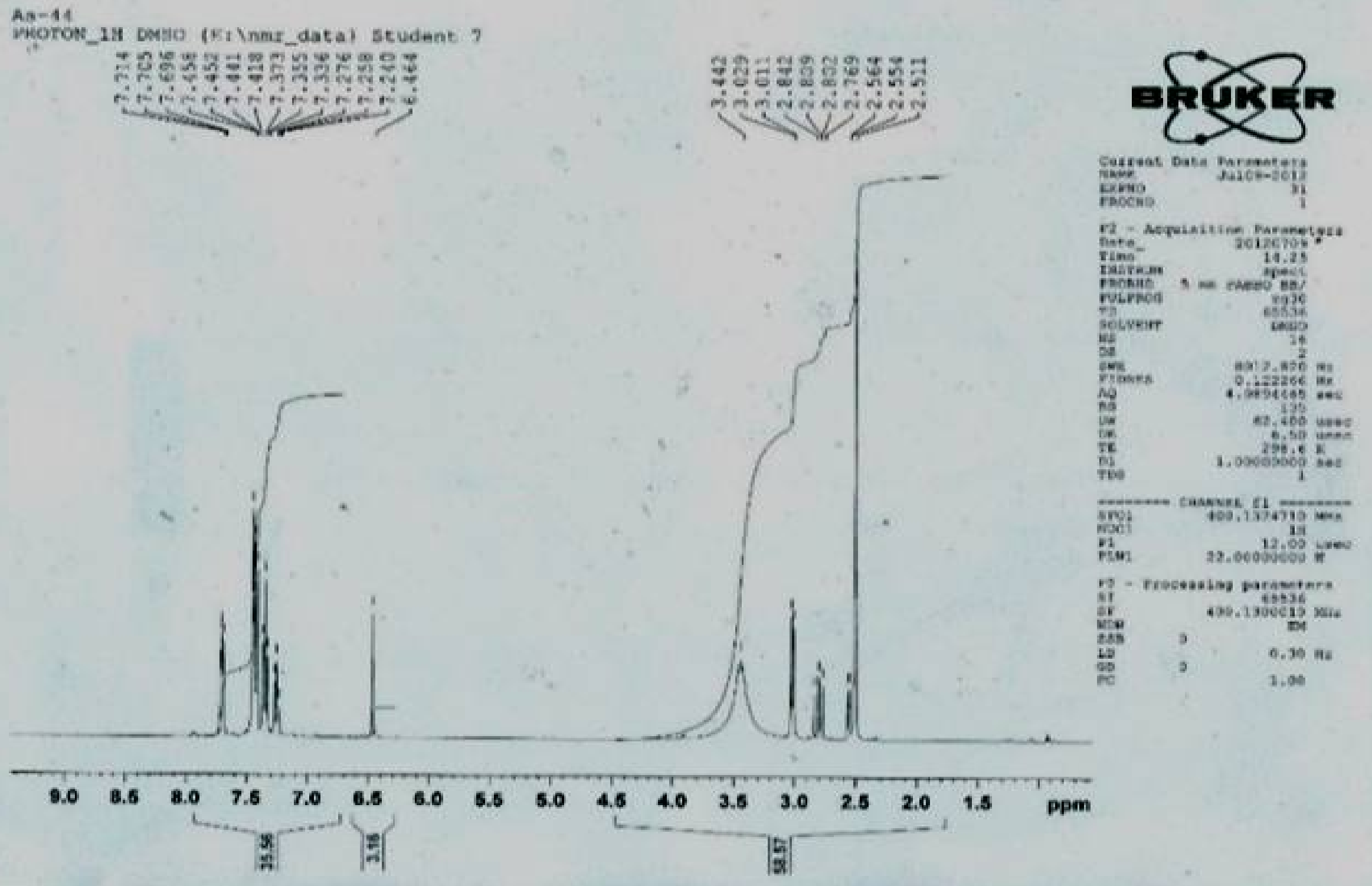


Figure S8. ${ }^{1} \mathrm{H}$ NMR (400 MHz, DMSO-d $\mathrm{d}_{6}$ ) of compound 6. 


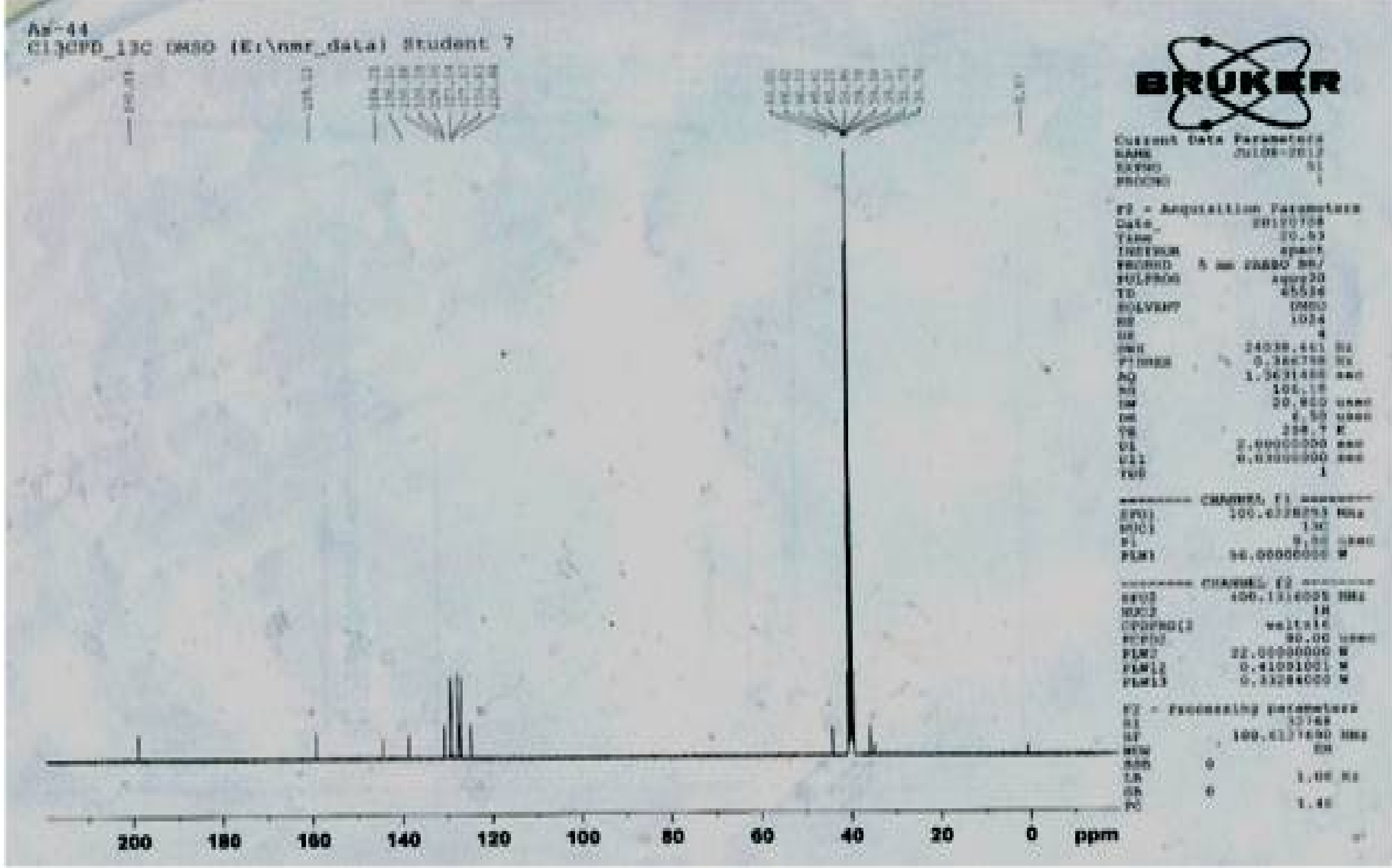


Figure S9. ${ }^{13} \mathrm{C}$ NMR (400 MHz, DMSO-d 6 ) of compound 6 . 


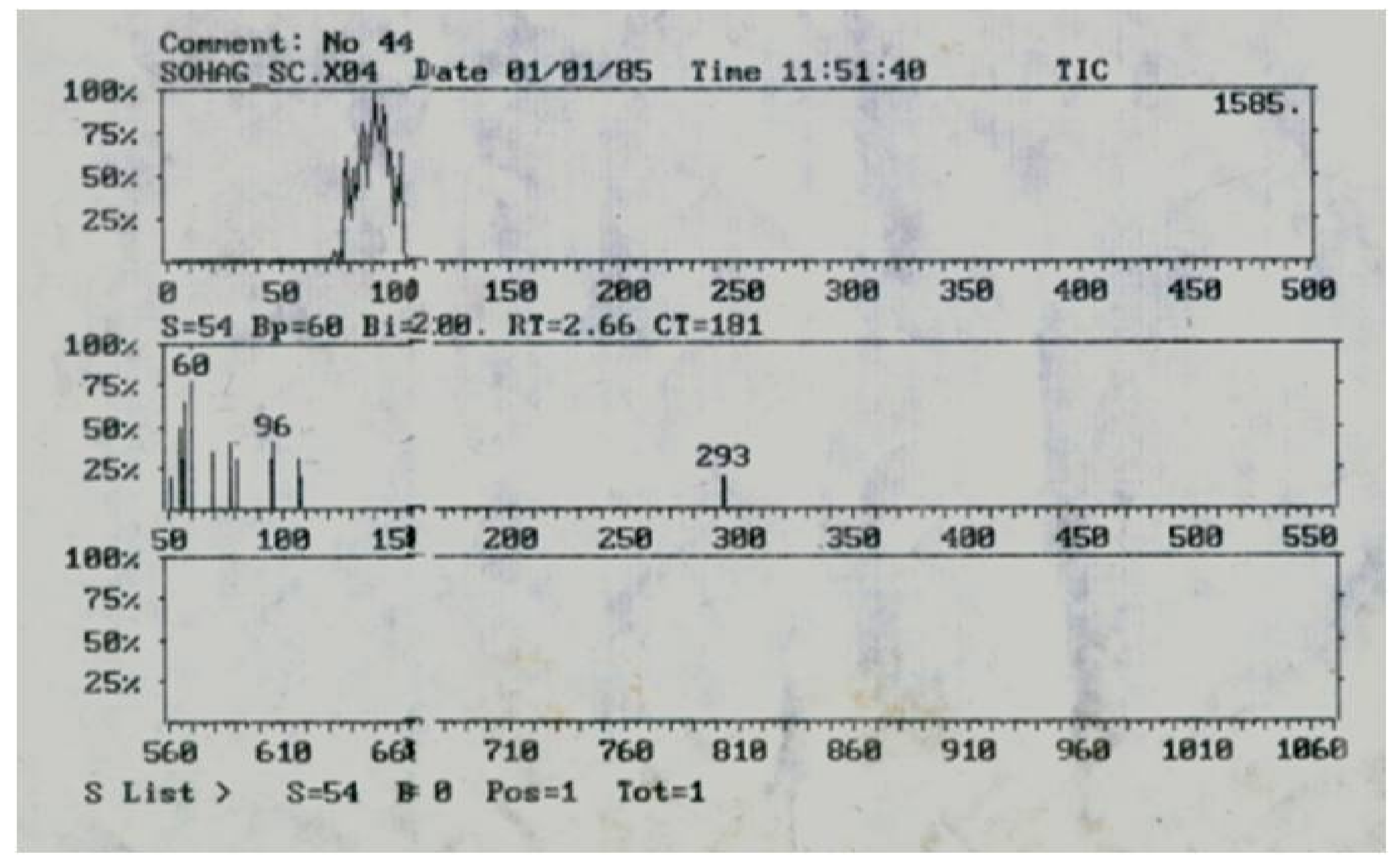




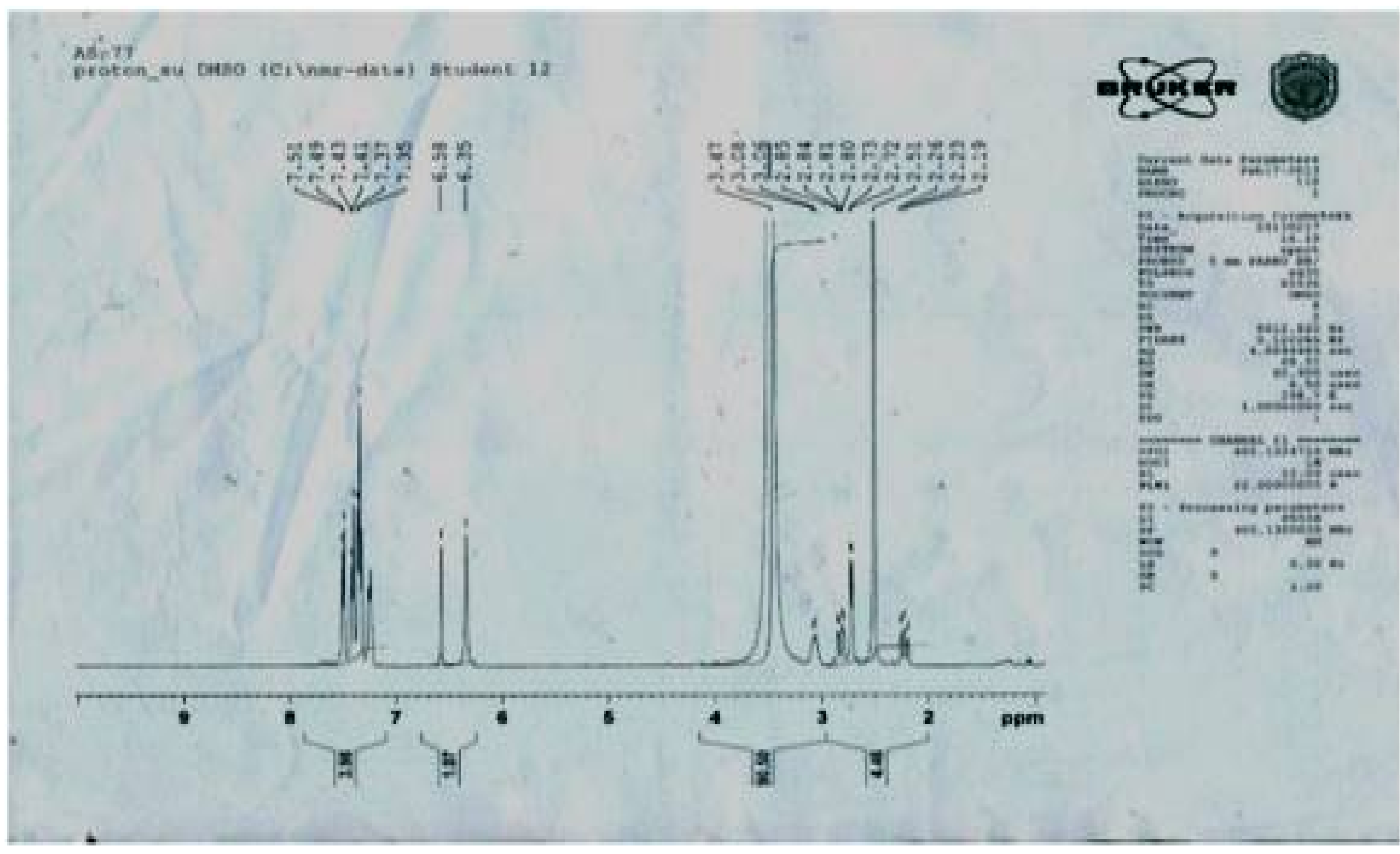


Figure S11. ${ }^{1} \mathrm{H}$ NMR (400 MHz, DMSO-d $\mathrm{d}_{6}$ ) of compound 7.

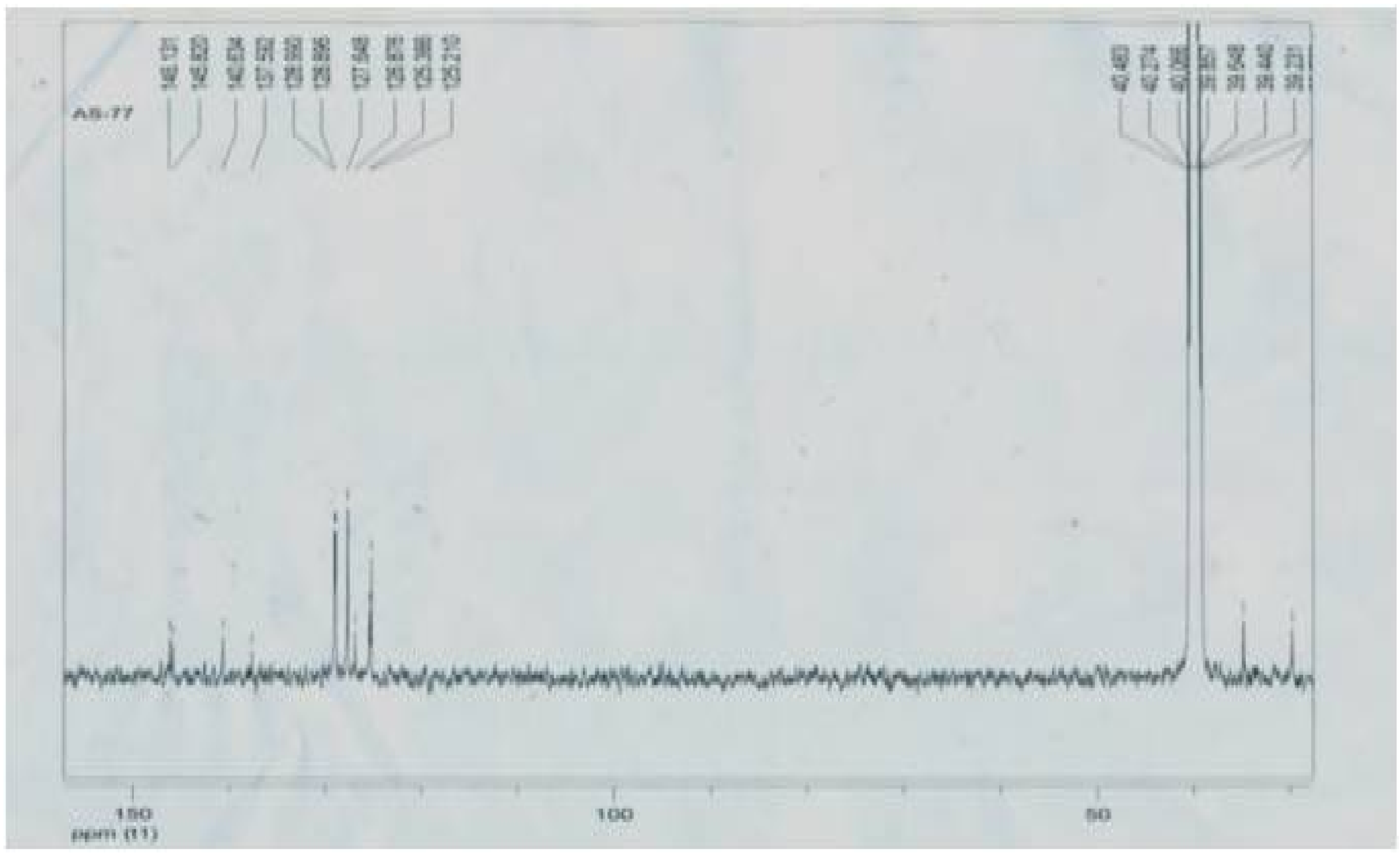


Figure S12. ${ }^{13} \mathrm{C}$ NMR (400 MHz, DMSO-d $\mathrm{d}_{6}$ ) of compound 7.

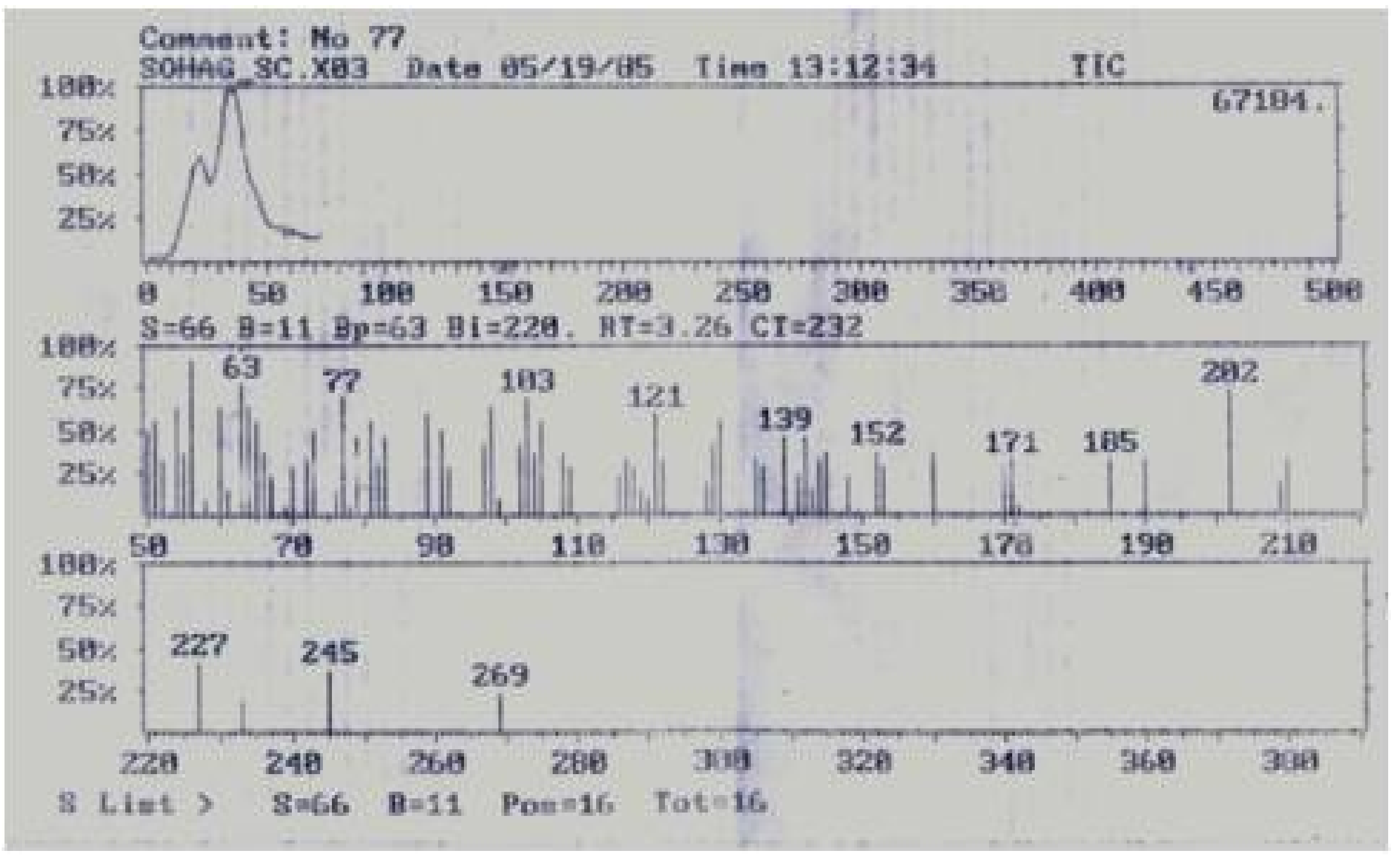


Figure S13. Mass spectra of compound 7.

\section{As-11s}

proton us cncis (C: hame-data) student 3

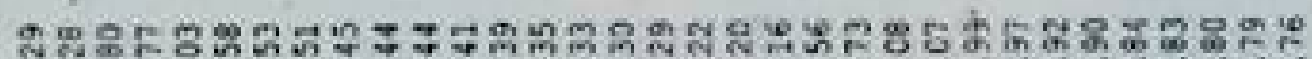

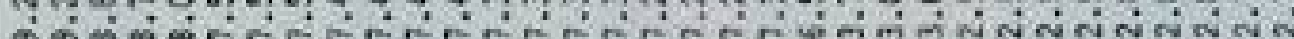

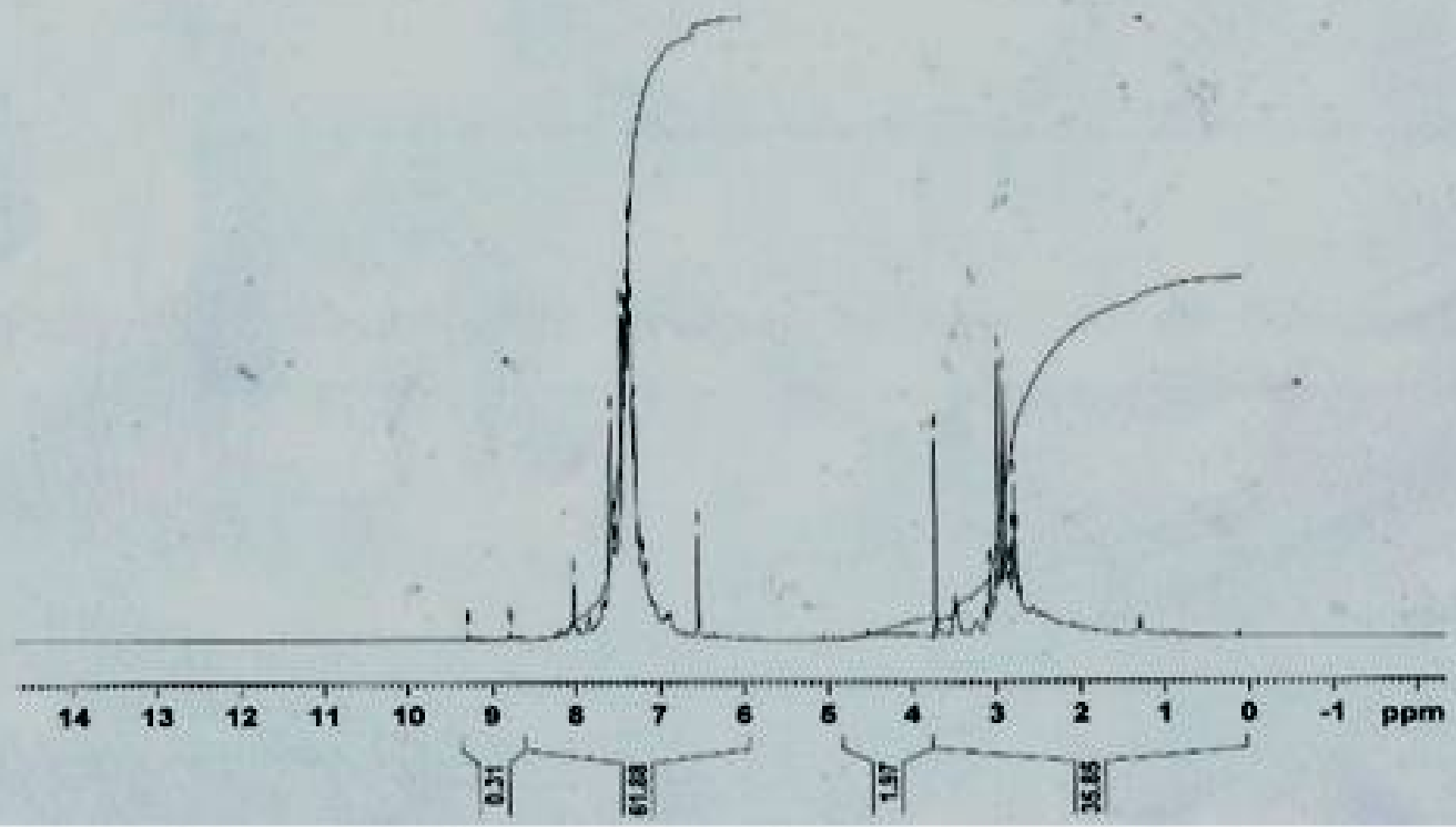

\section{$=\overbrace{1}\}_{n}$}

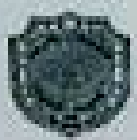

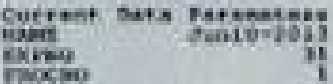

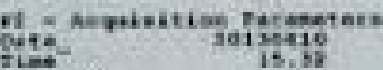

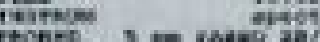

tints

nim

in.

inim

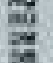

h.

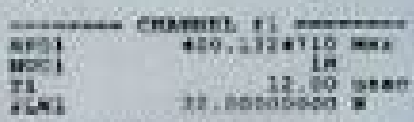

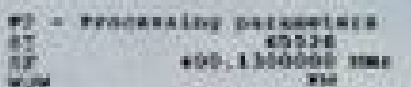

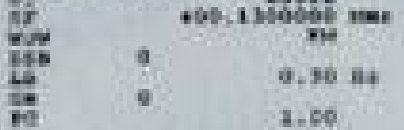


Figure S14. ${ }^{1} \mathrm{H}$ NMR $\left(400 \mathrm{MHz}, \mathrm{CCL}_{3}\right)$ of compound 12.

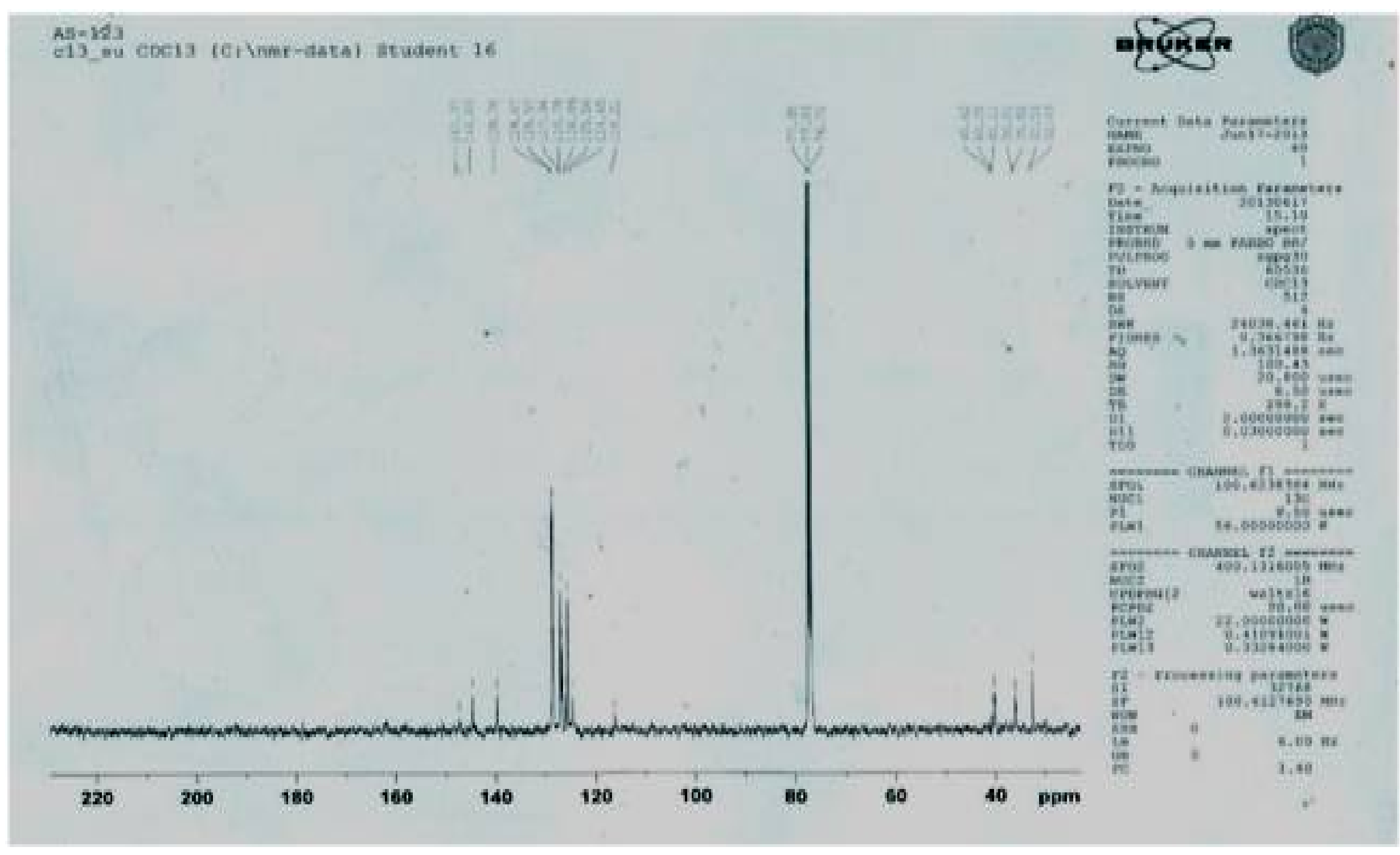


Figure S15. ${ }^{13} \mathrm{C}$ NMR (400 MHz, $\mathrm{CCL}_{3}$ ) of compound 12. 


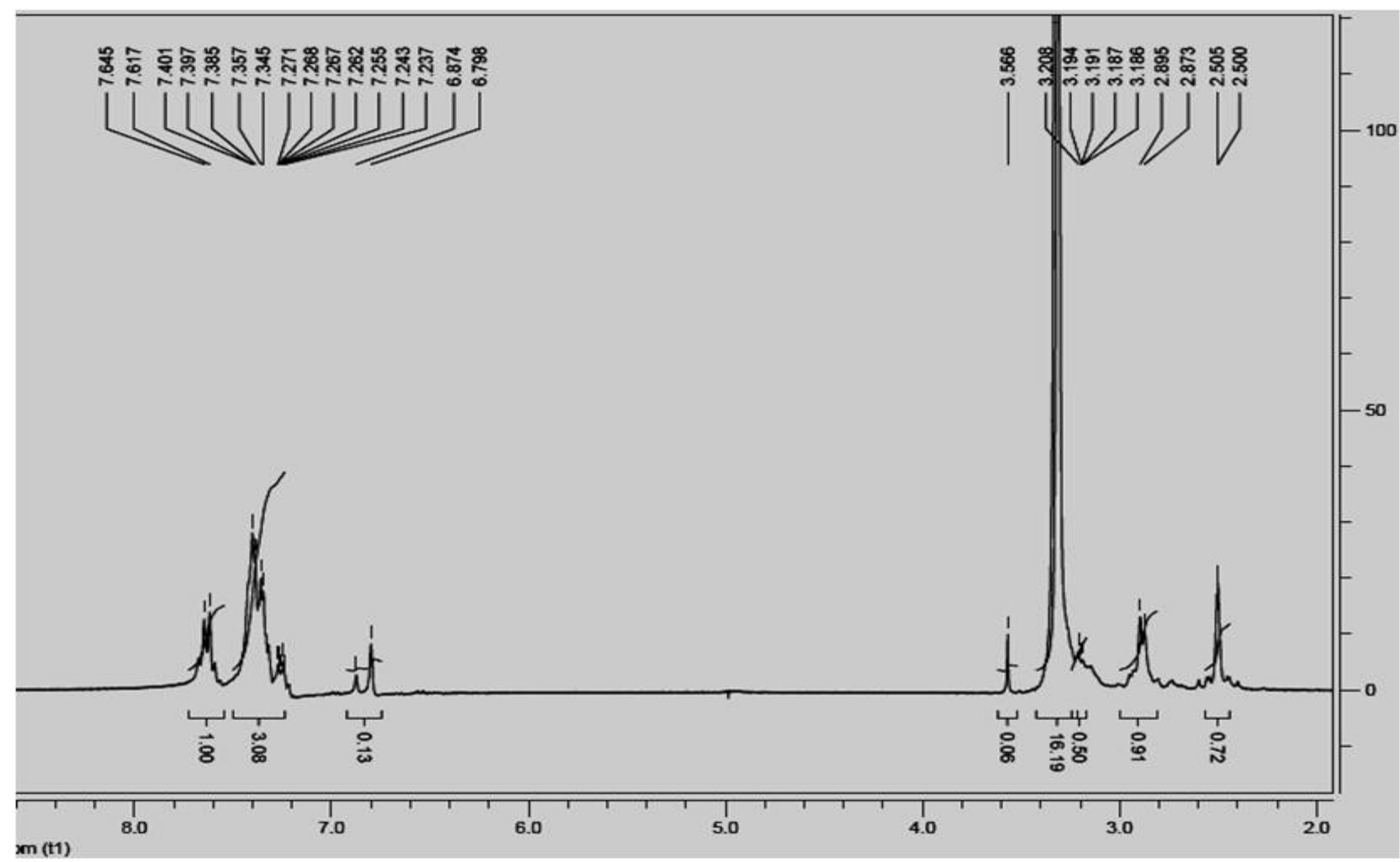


Figure S16. ${ }^{1} \mathrm{H}$ NMR $\left(400 \mathrm{MHz}, \mathrm{CCL}_{3}\right)$ of compound 15. 
AS -87

c13 su $\operatorname{CDC} 13$ \{C: $\backslash \mathrm{nmr}$-data\} Student 16

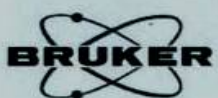

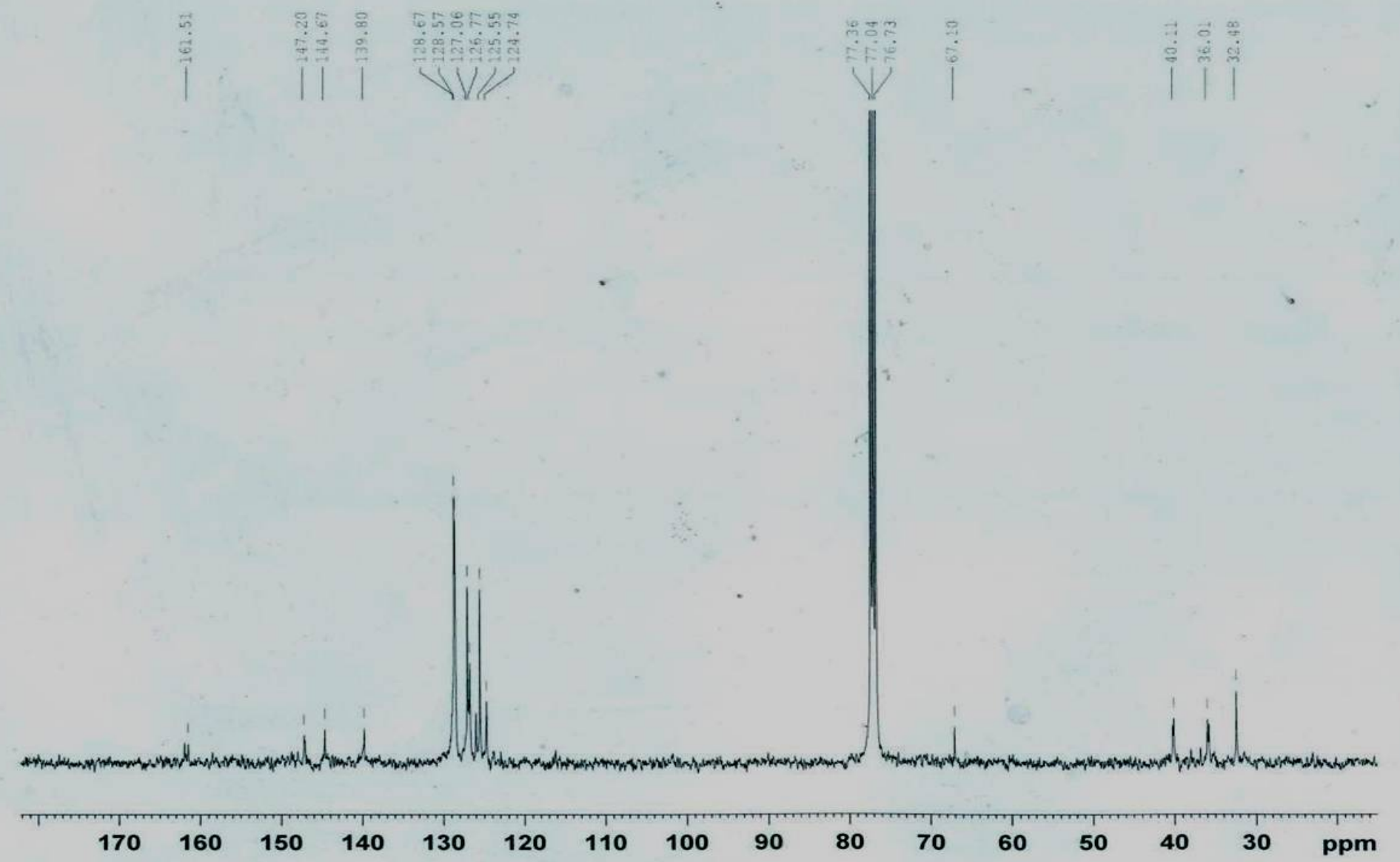


Figure S17. ${ }^{13} \mathrm{C}$ NMR $\left(400 \mathrm{MHz}, \mathrm{CCL}_{3}\right)$ of compound 15.

As:-128

proton_ou coci3 (C: \nenodate) atudent 15
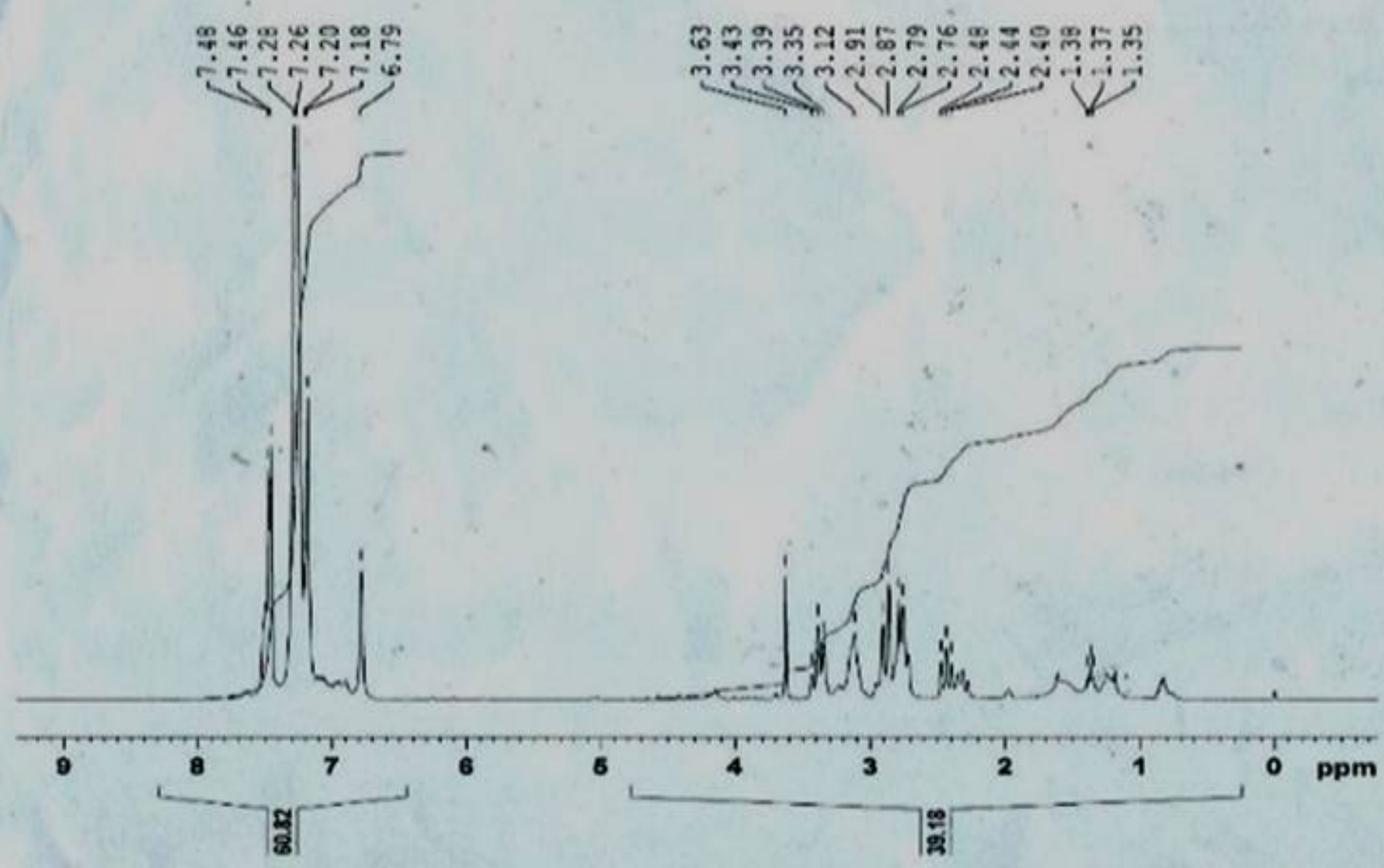

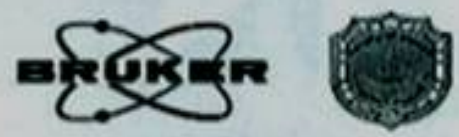
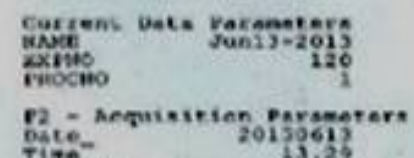

o4te- 201506?

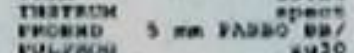

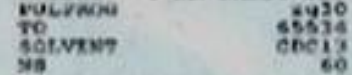

on.

$\min _{\operatorname{mon}}$

䠇

rol

sayzizon $\mathrm{mi}$

1. atopics

1. 0000000 b

พ701

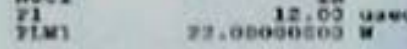

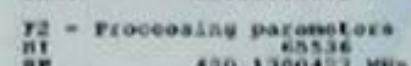

紫然

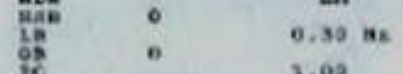

Figure S18. ${ }^{1} \mathrm{H}$ NMR $\left(400 \mathrm{MHz}, \mathrm{CCL}_{3}\right)$ of compound 18. 


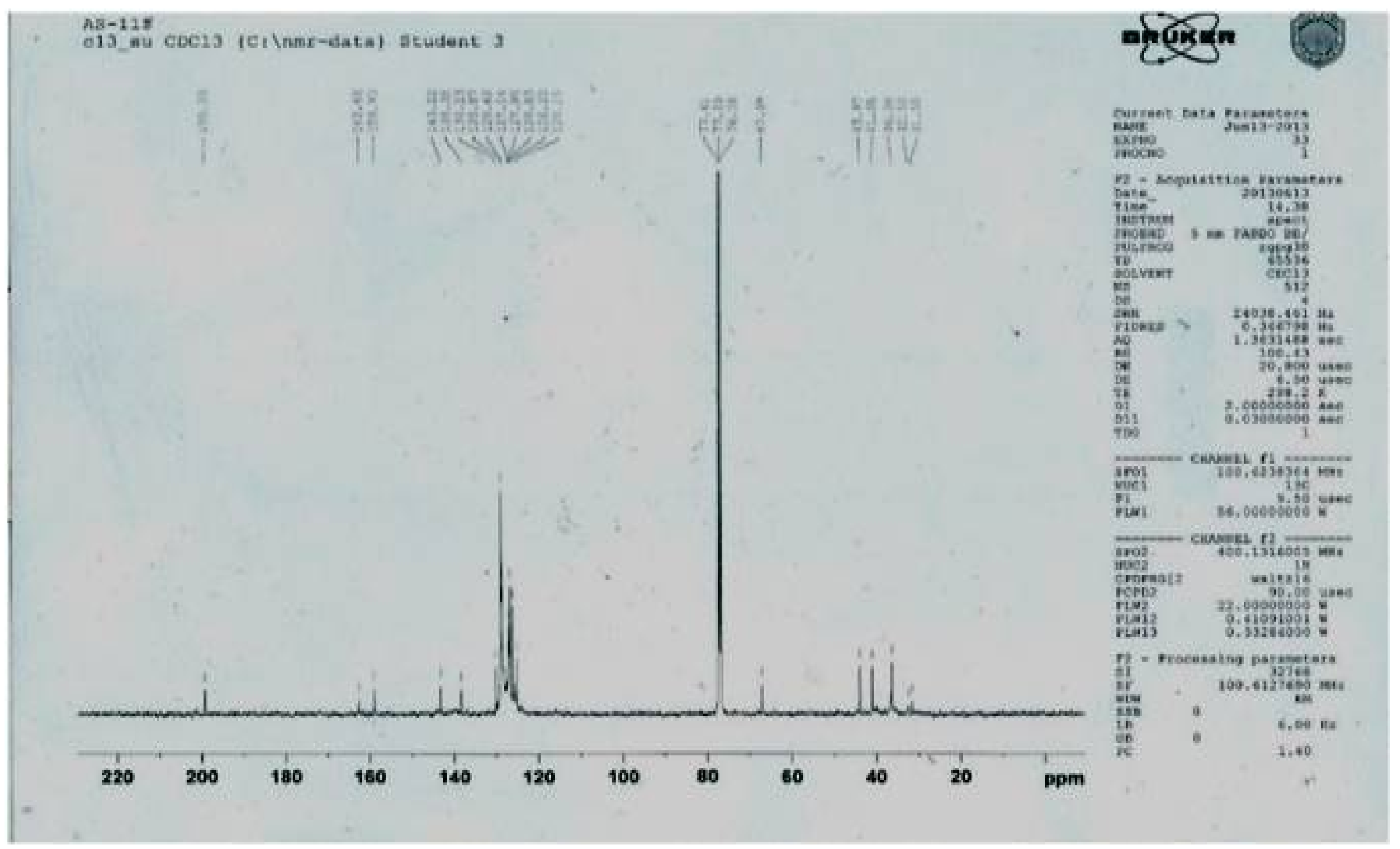


Figure S19. ${ }^{13} \mathrm{C}$ NMR (400 MHz, $\mathrm{CCL}_{3}$ ) of compound 18.

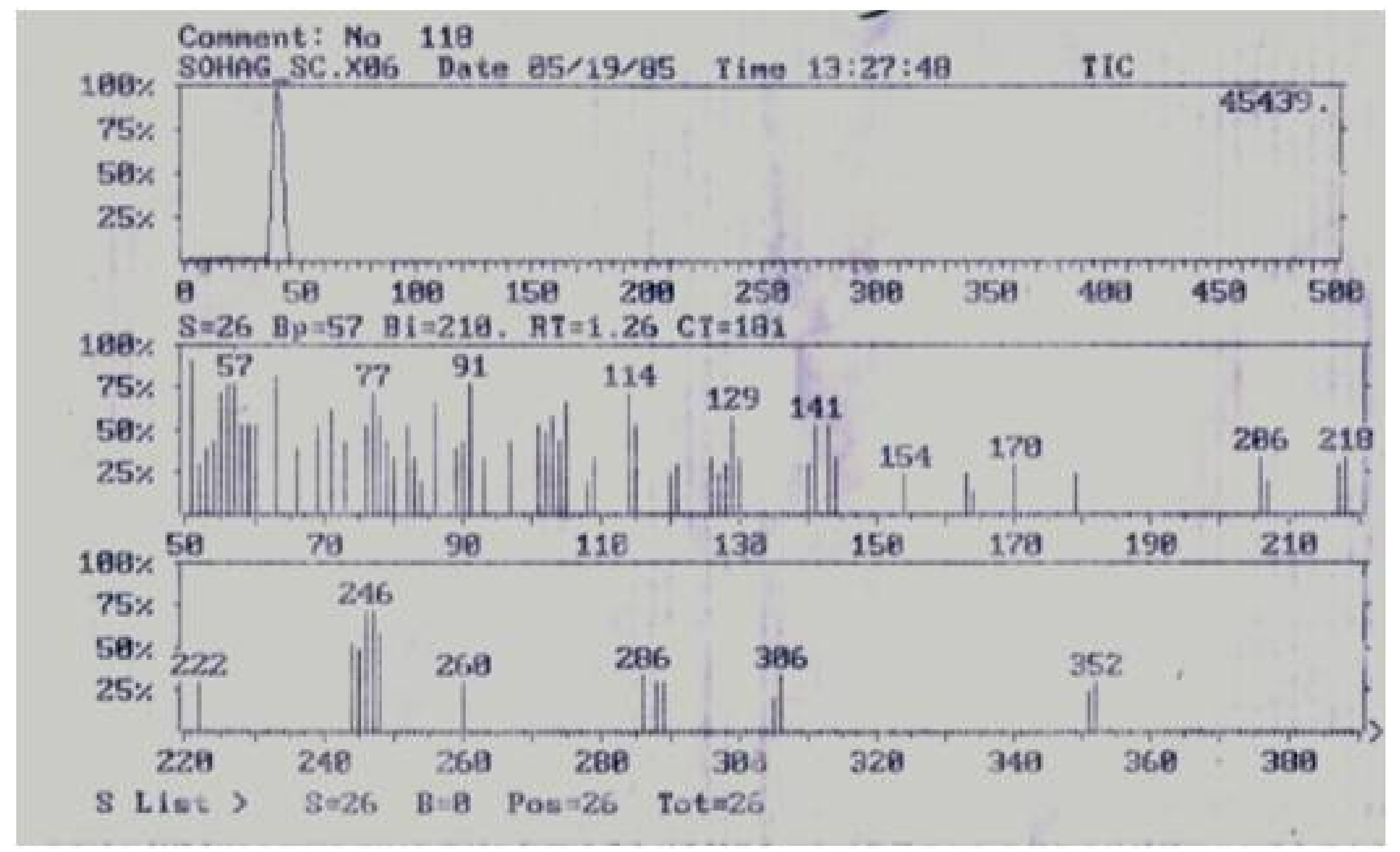


Figure S20. Mass spectra of compound 18. 